\title{
One-harmonic maps on Riemann surfaces
}

\author{
Stefano Trapani and Giorgio Valli
}

In this paper we study the properties of an "antiholomorphic energy functional" on a compact Riemann surface $R$. An interesting feature of this functional, not shared by the usual energy of harmonic maps, is its symmetry with respect to the inversion of the map. For surfaces of negative curvature we prove that, in any homotopy class containing an orientation-preserving diffeomorphisms, there exist a unique critical point. We also relate the functional to lagrangian area minimizing maps, as well as to Teichmüller theory and the abelian vortex equation. The functional is then seen to be related to the "distance" of an invariant Lorentzian metric. We will explicitly write the exponential coordinates of such metric. In these coordinates the Euler-Lagrange equation of the functional turns out to be linear, it also coincides with the equation of orthogonality to the orbits of the group $\mathcal{D}$ of diffeomorphism of the surface. The Lorentzian metric restricted to $\mathcal{M}_{-1} / \mathcal{D}$ is the Weil-Petersson metric, this allows us to express the equation of Weil-Petersson geodesics only in terms of the hyperbolic geometry of the surface.

\section{Introduction.}

Let $R$ be a compact connected Riemann surface of genus bigger then one. Let $\phi$ be a smooth map of $R$ into itself, let $g, h$ be hermitian metrics on $R$, such that it is endowed with the conformal structures which they induce. Consider the functional

$$
T_{\partial}(g, h, \phi)=\int_{R}\|\partial \phi\| d V o l_{g} .
$$

Where \|\| denotes the metric norm of $\partial \phi$. One might consider as well the analogous functional with the $\bar{\partial}$ and with the $\mathrm{d}$ operator. This is the $L^{1}$ version of the usual energy functionals

$$
E_{\partial}(g, h, \phi)=\int_{R}\|\partial \phi\|^{2} d V o l_{g}
$$




$$
\begin{aligned}
& E_{\bar{\partial}}(g, h, \phi)=\int_{R}\|\bar{\partial} \phi\|^{2} d V o l_{g} \\
& E_{d}(g, h, \phi)=\int_{R}\|d \phi\|^{2} d V o l_{g} .
\end{aligned}
$$

which for harmonic diffeomorphisms are related by the simple equations

$$
\begin{aligned}
& \frac{E_{d}}{2}=E_{\partial}+E_{\bar{\partial}} \\
& E_{\partial}-E_{\bar{\partial}}=\operatorname{Vol}_{h}(R) .
\end{aligned}
$$

The above energy functional have been studied by many people see for example [3], [10]. In particular it has been proved that, if $h$ has strictly negative Gauss curvature, then in any homotopy class of smooth maps from $\mathrm{R}$ to itself, there exist a unique critical point which is also smooth. Moreover if the homotopy class contains a diffeomorphism, then the critical point is itself a diffeomorphism, see [10]. Such critical points are known as harmonic maps. Critical points of the functionals $T_{\partial}, T_{\bar{\partial}}$, and $T_{d}$, seem to have different behaviour. However they share a symmetry property which is not enjoyed by harmonic maps, namely if $\phi$ is a diffeomorphism, then $T_{\partial}(g, h, \phi)=T_{\partial}\left(h, g, \phi^{-1}\right)$. The same holds for the functional, $T_{\bar{\partial}}$, and $T_{d}$. In this paper we will study $T_{\partial}$. We will show that, if the curvatures of $g$ and $h$ are both negative, then in any homotopy class, containing an "orientation preserving" diffeomorphism, there exists a unique critical map of $T_{\partial}$. Such map preserves the curvature form. Let us denote with $\mathcal{M}$ the space of hermitian metrics on $R$, with $\mathcal{M}_{-1}$ the ones with constant curvature -1 , with $\mathcal{D}$ the group of orientation preserving diffeomorphism and with $\mathcal{D}_{0}$ its identity component. In section 3 we introduce coordinates $(P, Q)$ on $\mathcal{M}$ which linearize the Euler-Lagrange equation of $T_{\partial}$. We also show that, for metrics of negative curvature, the orientation preserving critical diffeomorphisms of $T_{\partial}$ have a graph which is a minimal lagrangian submanifold in $R \times R$ with a suitable Riemannian metric. Section 4 is devoted to the special case where both $g$ and $h$ belong to $\mathcal{M}_{-1}$. The non antiholomorphic critical points are then simplectic. In this case the existence and uniqueness Theorem for critical points of $T_{\partial}$ follows from [10], and [12]. In light of this we can also relate the functional to the standard energy as a map from Teichmüller space into the reals, mainly studied by Tromba and Wolf, see [13], [14]. In section 5 we deal with an invariant generalized Lorentzian metric whose exponential coordinates coincide with $(P, Q)$. The Lorentzian "distance" on $\mathcal{M}$ can be expressed in terms of $T_{\partial}$, and the Euler-Lagrange equation of $T_{\partial}$ coincides with the equation of orthogonality to the $\mathcal{D}_{0}$ orbits. We also show that the medium point $m$ of the Lorentzian geodesics in 
$\mathcal{M} / \mathcal{D}_{0}$ between two points $g$ and $h$ in $\mathcal{M}_{-1} / \mathcal{D}_{0}$, is such that the diagonal map from $(R, m)$ into $(R, g) \times(R, h)$ is harmonic and conformal, moreover $(R, m)$ satisfies the abelian vortex equation, see [6]. The Lorentzian metric restricted to $\mathcal{M}_{-1}$ is positive definite, and the induced metric on Teichmüller space $\mathcal{T}=\mathcal{M}_{-1} / \mathcal{D}_{0}$ coincides with the Weil-Petersson metric. This allows us to determine the equation of the Weil-Petersson geodesics only in terms of the hyperbolic geometry of the surface. In the last section we prove the existence and uniqueness Theorem for critical points of $T_{\partial}$ homotopic to the identity. To obtain this result we use a Wente-type estimate, the Mumford, and Cheger-Gromov compactness Theorems, (see [2], [9], and [8]) and apply the "continuity method". The authors would like to thank professor G. Anzellotti, L. Simon, R. Schoen and the referee for many discussions and helpful suggestions. This work was completed while the second author was visiting Brest and Stanford University, he would like to express his gratitude for a very warm hospitality.

\section{The functional.}

Let $\mathrm{R}$ be a compact connected Riemann surface of genus strictly bigger then 1. Let $g, h$ be hermitian metrics on $\mathrm{R}$, and $\phi: R \rightarrow R$ be a smooth map of $R$ into itself; let us consider the functional

$$
T_{\partial}(g, h, \phi)=\int_{R}\|\partial \phi\| d V o l_{g}
$$

In an analogous way one can define the functional $T_{\bar{\partial}}$ and $T_{d}$. Let us give to $R$ the Riemann surface structures given by $g$ and $h$ respectively, then in holomorphic local coordinate we can write

$$
g=\rho^{2} d z d \bar{z}, h=\sigma^{2} d w d \bar{w} .
$$

Lemma 2.1. Let $\psi$ be an orientation preserving diffeomorphism of $R$, and $\phi$ a smooth map of $R$ into itself, then we have:

(i) If $\phi$ is a diffeomorphism then $T_{\partial}(g, h, \phi)=T_{\partial}\left(h, g, \phi^{-1}\right)$

(ii) $T_{\partial}\left(g, \psi^{*}(h), \phi\right)=T_{\partial}(g, h, \phi \circ \psi)$

(iii) $T_{\partial}\left(a^{2} g, h, \phi\right)=a T_{\partial}(g, h, \phi)$ for every positive constant $a$.

(iv) If $g=h$ then the identity map is a critical point of $T_{\partial}$.

The same relations hold for $T_{\bar{\partial}}$ and for $T_{d}$. 
Proof. Let us prove (i) In holomorphic local coordinates we have

$$
\left\|\partial \phi^{-1}\right\|=\frac{\left|\frac{\partial \phi^{-1}}{\partial w}\right| \rho\left(\phi^{-1}(w)\right)}{\sigma(w)} .
$$

Since, by Cramer role for linear systems we have

$$
\left|\left(\frac{\partial \phi^{-1}}{\partial w}\right)(\phi)\right|=\frac{\left|\frac{\partial \phi}{\partial z}\right|}{|\operatorname{det}(J a c(\phi))|}
$$

in the case of $T_{\partial}$, we conclude by change of variable via the diffeomorphism $\phi$. Here Jac is the jacobian matrix. The case of $T_{\bar{\partial}}$ is similar.

In the case of $T_{d}$ we have in real coordinates

$$
\left\|d\left(\phi^{-1}\right)\right\|=\sqrt{\frac{\rho^{2}\left(\phi^{-1}\right)}{\sigma^{2}} \operatorname{trace}\left(\operatorname{Jac}\left(\phi^{-1}\right)^{T} J a c\left(\phi^{-1}\right)\right)} .
$$

Hence the claim follows from change of variable in the integral via the diffeomorphism $\phi$, together with the identity

$$
\sqrt{\left(\operatorname{trace}\left(\operatorname{Jac}(\phi) \operatorname{Jac}(\phi)^{T}\right)^{-1}\right.}|\operatorname{det}(\operatorname{Jac}(\phi))|=\sqrt{\left(\operatorname{trace}\left(\operatorname{Jac}(\phi) \operatorname{Jac}(\phi)^{T}\right)\right.},
$$

which can be proved by using the eigenvalues of the positive definite symmetric two by two real matrix $J a c(\phi) J a c(\phi)^{T}$.

The properties (ii) and (iii) are clear.

For (iv) we may consider a variation $\phi_{t}$ of $\phi$, differentiate the functional with respect to $t$, and compute at $t=0$. Since, denoting the identity map with I, we have $\|\bar{\partial}(I)\| \equiv 0,\|\partial(I)\|=\|d(I)\| \equiv 1$, and the identity is harmonic, we conclude.

In this paper we will only study the functional $T_{\partial}$. The Euler-Lagrange operator $\mathcal{E}(\phi, g, h)$ for the functional $T_{\partial}$ is given in local holomorphic coordinates by:

$$
\begin{array}{r}
\mathcal{E}(\phi, g, h)=\rho \sigma(\phi)\left(\partial \log \left(\frac{\partial \phi}{\overline{\partial \phi}}\right)+\left(\partial_{w} \log \sigma^{2}\right)(\phi)(\partial \phi)\right) \frac{\overline{\partial \phi}}{|\partial \phi|} \\
-\rho \sigma(\phi))\left(\left(\bar{\partial}_{w} \log \sigma^{2}\right)(\phi) \partial \bar{\phi}\right) \frac{\overline{\partial \phi}}{|\partial \phi|} \\
-\rho \sigma(\phi)\left(\partial_{z}\left(\log \rho^{2}\right)\right) \frac{\overline{\partial \phi}}{|\partial \phi|}
\end{array}
$$


Note that if $\phi$ is an orientation preserving diffeomorphism, then

$$
|\partial \phi| \geq \sqrt{\mid \partial \phi)\left.\right|^{2}-|\bar{\partial} \phi|^{2}}>0,
$$

hence $\mathcal{E}(\phi, g, h)$ is well defined and smooth on the whose surface.

Let $\phi$ be a smooth map from $\mathrm{R}$ into itself, let $B=\{x \in R: \partial \phi(x) \neq 0\}$. We say that $\phi$ is a critical point of $T_{\partial}$ if $\mathcal{E}(\phi, g, h)(x)=0$ for every $\mathrm{x}$ in $\mathrm{B}$.

Lemma 2.2. If $\phi$ is a smooth critical point of $T_{\partial}$ then it preserves the curvature forms in every point of $B$, i.e. we have:

$$
K_{g} d V o_{g}(x)=\phi^{*}\left(K_{h} d V o_{h}\right)(x)
$$

for every $x \in B$. Where $K_{g}$ and $K_{h}$ are the Gauss curvatures of $g, h$ respectively.

Proof. Consider the operator

$$
\mathcal{E}^{\prime}(\phi, g, h)=\frac{\mathcal{E}(\phi, g, h)|\partial \phi|}{\rho \sigma(\phi) \overline{\partial \phi}} .
$$

Since $\phi$ is critical, then the real part of $\bar{\partial} \mathcal{E}^{\prime}$ vanishes identically, we will denote with $R e$ the real and with $I m$ the imaginary part of a complex number. We have:

$$
\begin{aligned}
& R e \bar{\partial} \mathcal{E}^{\prime}(\phi, h, g)=\bar{\partial}_{w} \partial_{w}\left(\log \rho^{2}\right)(\phi)\left(|\bar{\partial} \phi|^{2}-|\partial \phi|^{2}\right) \\
& +\bar{\partial} \partial\left(\log \sigma^{2}\right) .
\end{aligned}
$$

But $\bar{\partial} \partial\left(\log \rho^{2}\right)=-K_{g} \frac{\rho^{2}}{2}$ and $\bar{\partial} \partial\left(\log \sigma^{2}\right)=-K_{h} \frac{\sigma^{2}}{2}$. The result follows.

Remark 1. If $g$ and $h$ have negative curvature everywhere, then every smooth non antiholomorphic critical point of $T_{\partial}$, must be an orientation preserving diffeomorphism. In fact, by the above Lemma, since $\phi$ is smooth, then the jacobian determinant of $\phi$ is positive on the closure of $B$, hence $B$ is open and closed, so if it is non empty it must coincide with R. In particular if $K_{g} \equiv K_{h} \equiv-1$ then every non antiholomorphic critical point of $T_{\partial}$ is a simplectic diffeomorphism. Similarly, if $g$ has negative curvature everywhere and there exists a smooth critical point of $T_{\partial}$ which is an orientation preserving diffeomorphism, then $h$ has also negative curvature everywhere. 
Lemma 2.3. Let $g$ and $h$ be metrics on $R$ with negative curvature, let $\phi$ : $(R, g) \rightarrow(R, h)$ be an orientation preserving critical diffeomorphism of $T_{\partial}$. We have that the second variation of $T_{\partial}$ at $\phi$ is positive definite.

Proof. Recall that

$$
\mathcal{E}=\frac{\mathcal{E}^{\prime} \rho \sigma(\phi) \overline{\partial \phi}}{|\partial \phi|} .
$$

Hence if $\phi$ is critical, then

$$
\frac{\partial \mathcal{E}}{\partial t}_{\mid t=0}=\frac{{\frac{\partial \mathcal{E}^{\prime}}{\partial t}}_{\mid t=0} \rho \sigma(\phi) \overline{\partial \phi}}{|\partial \phi|} .
$$

If $u$ is a non zero vector field on $R$ and $L$ the linearized of the operator $\mathcal{E}^{\prime}$ we have

$$
\begin{gathered}
L(u)=\partial\left(\frac{\partial u}{\partial \phi}-\frac{\bar{\partial} \bar{u}}{\bar{\partial} \bar{\phi}}\right) \\
+\partial_{w}{ }^{2}\left(\log \sigma^{2}\right)(\partial \phi) u-\bar{\partial}_{w}^{2}\left(\log \sigma^{2}\right)(\partial \bar{\phi})(\bar{u}) \\
+\partial_{w} \bar{\partial}_{w}\left(\log \sigma^{2}\right)(\bar{u} \partial \phi-u \bar{\partial} \phi)+\partial_{w}\left(\log \sigma^{2}\right) \partial u-\bar{\partial}_{w}\left(\log \sigma^{2}\right) \partial \bar{u}
\end{gathered}
$$

In other words

$$
\begin{gathered}
L(u)=\partial\left(\left(\frac{\partial u}{\partial \phi}+\partial_{w}\left(\log \sigma^{2}\right) u\right)-\left(\frac{\bar{\partial} \bar{u}}{\bar{\partial} \bar{\phi}}+\bar{\partial}_{w}\left(\log \sigma^{2}\right) \bar{u}\right)\right) \\
+2 \partial_{w} \bar{\partial}_{w}\left(\log \sigma^{2}\right)(\bar{u} \partial \phi-u \partial \bar{\phi}) .
\end{gathered}
$$

If we set

$$
v=\frac{\bar{u}}{\bar{\partial} \bar{\phi}}
$$

and

$$
\mathcal{F}=\rho \sigma(\phi)|\partial \phi|
$$

the above equation becomes

$$
\begin{gathered}
L(u)=\partial\left(\partial \bar{v}+\bar{v}\left(\partial_{w}\left(\log \sigma^{2}\right) \partial \phi+\partial(\log \partial \phi)\right)\right) \\
-\partial\left(\bar{\partial} v+v\left(\bar{\partial}_{w}\left(\log \sigma^{2}\right) \bar{\partial} \bar{\phi}+\bar{\partial}(\log \bar{\partial} \bar{\phi})\right)\right) \\
\left.+2 \partial_{w} \bar{\partial}_{w}\left(\log \sigma^{2}\right)\left(v|\partial \phi|^{2}-\bar{v} \partial \phi \partial \bar{\phi}\right)\right) .
\end{gathered}
$$

Set

$$
\gamma=\mathcal{F} v=\frac{\bar{u} \sigma(\phi) \rho|\partial \phi|}{\bar{\partial} \bar{\phi}}
$$


and $\omega=\gamma d z+\bar{\gamma} d \bar{z}$, we may think of $L$ as a function of $\omega$, and using the Euler-Lagrange equation we find

$$
L(\omega)=\partial\left(\frac{1}{\mathcal{F}}(\partial \bar{\gamma}-\bar{\partial} \gamma)\right)+2 \partial_{w} \bar{\partial}_{w}\left(\log \sigma^{2}\right) \frac{\partial \phi}{\mathcal{F}}(\gamma \bar{\partial} \bar{\phi}-\bar{\gamma} \bar{\partial} \phi) .
$$

The second variation of $T_{\partial}$ is given by

$$
2 \int_{R} \operatorname{Re}\left(L(u) u \frac{\sigma(\phi) \bar{\partial} \bar{\phi}}{\rho|\partial \phi|}\right) d V o l_{g}=2 \int_{R} \operatorname{Re}(L(\omega) \bar{\gamma})(i / 2) d z \wedge d \bar{z}
$$

Hence by adding formula (2.1) with its conjugate, and integrating by part we find

$$
\begin{aligned}
& {\frac{\partial^{2} T_{\partial}}{\partial t^{2}}}_{\mid t=0}=\int_{R} \frac{-1}{\mathcal{F}}(\partial \bar{\gamma}-\bar{\partial} \gamma)^{2}(i / 2) d z \wedge d \bar{z} \\
& +2 R e\left(\int_{R} \frac{-K_{h} \sigma^{2}(\phi)}{\mathcal{F}} \partial \phi(\gamma \bar{\partial} \bar{\phi}-\bar{\gamma} \partial \bar{\phi}) \bar{\gamma}(i / 2) d z \wedge d \bar{z}\right) .
\end{aligned}
$$

We have

$$
\begin{gathered}
2 \operatorname{Re}(\partial \phi(\gamma \bar{\partial} \bar{\phi}-\bar{\gamma} \partial \bar{\phi}) \bar{\gamma}) \\
=2|\gamma|^{2}|\partial \phi|^{2}\left(1-\operatorname{Re}\left(\frac{\gamma^{2}}{|\gamma|^{2}} \frac{\bar{\partial} \phi}{\partial \phi}\right)\right)>0
\end{gathered}
$$

since $\phi$ is an orientation preserving diffeomorphism.

Let us consider the function $S=-\phi^{*}\left(K_{h} d V o l_{h}\right) / d V o l_{g}+K_{g}$. By looking at the one-form $2 R e \mathcal{E}^{\prime} d z=\mathcal{E}^{\prime} d z+\overline{\mathcal{E}}^{\prime} d \bar{z}$, we find as in Lemma 2.2 that $d^{*}\left(2 \operatorname{Re} \mathcal{E}^{\prime}\right)=S$, where $d^{*}=* d *$ and $*$ is the Hodge $*$ operator with respect to $g$. Consider the operator $M=2 R e \mathcal{E}^{\prime} d z+d S$ then we have the following

Lemma 2.4. If $g$ and $h$ have negative curvature, and $\phi$ is an orientation preserving diffeomorphism, then the operator $M$ is elliptic and $\mathcal{E}^{\prime}(\phi)=0$ if and only if $M(\phi)=0$.

Proof. We know from Lemma 2.2 that if $\mathcal{E}^{\prime}=0$ then $M(\phi)=0$, viceversa if $M(\phi)=0$ then $\left(I+d d^{*}\right)\left(2 \operatorname{Re}\left(\mathcal{E}^{\prime}(\phi) d z\right)\right)=0$ which implies that $\mathcal{E}^{\prime}(\phi)=0$ since $I+d d^{*}$ is a positive operator. Now for a fixed non zero complex number $\xi$, set $\alpha=\frac{1}{\partial \phi}, \beta=\frac{-2 K_{h} \sigma^{2} \bar{\partial} \bar{\phi}}{\rho^{2}}$, and $\gamma=\frac{-2 K_{h} \sigma^{2} \partial \bar{\phi}}{\rho^{2}}$, then the top order symbol of $M$ is represented by the matrix

$$
A=\left(\begin{array}{cc}
(\alpha-\beta) \xi^{2}+\gamma|\xi|^{2} & -(\bar{\alpha}+\bar{\beta})|\xi|^{2}+\bar{\gamma} \xi^{2} \\
-(\alpha+\beta)|\xi|^{2}+\gamma \bar{\xi}^{2} & (\bar{\alpha}-\bar{\beta}) \bar{\xi}^{2}+\bar{\gamma}|\xi|^{2}
\end{array}\right)
$$


So

$$
\operatorname{det}(A)=-4|\xi|^{4}\left(\operatorname{Re}\left(\alpha \bar{\beta}-\frac{\alpha \bar{\gamma} \xi^{2}}{|\xi|^{2}}\right)\right)=8 K_{h}|\xi|^{4} \frac{\sigma^{2}}{\rho^{2}}\left(1-\operatorname{Re}\left(\frac{\xi^{2} \bar{\partial} \phi}{|\xi|^{2} \partial \phi}\right)\right)
$$

which is negative since $\phi$ is an orientation preserving diffeomorphism. Note that $|\operatorname{det}(A)| \geq \frac{-4 K_{h}|\xi|^{4} \sigma^{2} J a c(\phi)}{\rho^{2}|\partial \phi|^{2}}$. Hence when $\phi$ is $T_{\partial}$ critical, Lemma 2.2, gives $|\operatorname{det} A| \geq \frac{-4 K_{g}|\xi|^{4}}{|\partial \phi|^{2}}$.

\section{The coordinates $P, Q$.}

Let us now fix a square root $k^{1 / 2}$ of the canonical bundle of $\mathrm{R}$, let $\mathrm{r}$ be an integer, let $S(r)$ be the smooth sections of $k^{r / 2} \times \bar{k}^{-r / 2}$. For example the sections of $S(-2)$ are known as Beltrami differentials. Let $\mathcal{M}$ be the space of hermitian metrics on $R$. Let us fix an element $g \in \mathcal{M}$, and conformal coordinates on $\mathrm{R}$ where $\mathrm{g}$ is given as $g=\rho^{2} d z d \bar{z}$. We have a natural map from $S(0) \times S(-2)$ into $\mathcal{M}$ given by $\alpha(B, A)=\bar{A} \rho^{2} d z d \bar{z}+(2 B+1) \rho^{2} d z d \bar{z}+$ $A \rho^{2} d z d \bar{z}$. Then $\alpha(B, A)$ is a metric if and only if $B+1 / 2$ and $(B+1 / 2)^{2}-|A|^{2}$ are positive. Consider the open set $\Omega \subseteq S(0) \times S(-2)$ given by

$$
\Omega=\left\{(P, Q): P+1>0 \text { and }(P+1)^{2}-|Q|^{2}>0\right\} .
$$

We have the following

Proposition 3.1. The map $\Psi$ from $\Omega$ into $\mathcal{M}$ given by

$$
\left.\Psi(P, Q)=\left((P+1) \bar{Q}+\left((P+1)^{2}+|Q|^{2}\right)+(P+1) Q\right)\right) g
$$

is an open embedding.

Proof. In coordinate $(B, A)$ the map reads

$$
\Psi(P, Q) \rightarrow\left(\frac{\left.(P+1)^{2}+|Q|^{2}\right)-1}{2},(P+1) Q\right),
$$

whereas we have

$$
\begin{aligned}
\Psi^{-1} & (B, A) \\
& =\left(\sqrt{\frac{2 B+1+\sqrt{(2 B+1)^{2}-4|A|^{2}}}{2}}-1, \frac{A}{\sqrt{\frac{2 B+1+\sqrt{(2 B+1)^{2}-4|A|^{2}}}{2}}}\right)
\end{aligned}
$$


and these are both smooth maps. Note that, after having identified the tangent space to $\mathcal{M}$ at $g$ with $S(0) \times S(-2)$ via the map $\alpha$, we find that $\Psi(0,0)=g$ and $d \Psi(0,0)=I$.

Denote by $P_{h}, Q_{h}$, the coordinates of the metric $h$ given by the above Proposition. Set for convenience $F_{h}=P_{h}+1$. Let $\phi: R \rightarrow R$ be a smooth map, and h a smooth metric, set $\alpha=\frac{\partial \phi}{\partial z}$ and $\beta=\frac{\partial \phi}{\partial \bar{z}}, F_{\phi^{*}(h)}=F$ and $Q_{\phi^{*}(h)}=\tilde{Q} d \bar{z} / d z$. Then $F=\frac{\sigma|\alpha|}{\rho}$ and $\tilde{Q}=\frac{\sigma \bar{\alpha} \beta}{\rho|\alpha|}$. So

$$
\partial\left(\log F^{2}\right)=\partial(\log \alpha)+\partial(\log \bar{\alpha})+\partial_{w}\left(\log \sigma^{2}\right) \alpha+\partial_{\bar{w}}\left(\log \sigma^{2}\right) \bar{\beta}-\partial\left(\log \rho^{2}\right) .
$$

Hence the Euler-Lagrange equation $\mathcal{E}^{\prime}=0$ is equivalent to

$$
\partial(\log F)=\partial(\log \bar{\alpha})+\partial_{\bar{w}}\left(\log \sigma^{2}\right) \bar{\beta} .
$$

Observe that $\bar{\partial} \alpha=\partial \beta$. Define $M=\sigma^{2} \bar{\alpha} \beta=\rho^{2} F \tilde{Q}$. We have

$$
\partial(\log (M))=\partial\left(\log \sigma^{2}\right)+\partial(\log \bar{\alpha})+\frac{\bar{\partial} \alpha}{\beta} .
$$

However $\frac{\alpha}{\beta}=\frac{F}{\tilde{Q}}$ so

$$
\partial(\log (M))=\partial\left(\log \sigma^{2}\right)+\partial(\log \bar{\alpha})+\bar{\partial}(\log \alpha) \frac{F}{\tilde{Q}} .
$$

And by plugging (3.1) and its conjugate into the above equation we find

$$
\partial \log M=\partial \log \sigma^{2}+\partial \log F-\bar{\partial}_{w} \log \sigma^{2} \bar{\beta}-\frac{F}{\tilde{Q}} \partial_{w} \log \sigma^{2} \beta+\frac{F}{\tilde{Q}} \bar{\partial} \log F
$$

i.e.

$$
\partial \log F+\partial \log \tilde{Q}+\partial \log \rho^{2}=\partial \log M=\partial \log F+\frac{F \bar{\partial} \log F}{\tilde{Q}}
$$

that is

$$
\frac{\partial \tilde{Q}}{\tilde{Q}}+\partial \log \rho^{2}-\frac{\bar{\partial} F}{\tilde{Q}}=0
$$

or

$$
\rho^{-3} \partial\left(\rho^{2} \tilde{Q}\right)=\rho^{-1} \bar{\partial} F .
$$

There are natural global differential operators on $(R, g)$,

$$
\begin{aligned}
& L_{r}: S(r) \rightarrow S(r-1) \\
& K_{r}: S(r) \rightarrow S(r+1)
\end{aligned}
$$


(essentially the covariant complex derivatives) defined as follows:

$$
\begin{aligned}
& K_{r}(a)=\rho^{r-1} \partial\left(\rho^{-r} a\right) \\
& L_{r}(a)=\rho^{-r-1} \bar{\partial}\left(\rho^{r} a\right)
\end{aligned}
$$

The main properties of the operators $L_{r}, K_{r}$ are the following

$$
\begin{aligned}
& K_{r}=\overline{L_{-r}} \\
& \Delta_{r}=4 L_{r+1} K_{r}+r(r+1) I=\quad \text { where } \Delta_{r} \text { is the } \partial \text { laplacian on } S_{r} . \\
& L_{r+1} K_{r}=K_{r-1} L_{r}+(r / 2) K_{g} \\
& \int_{R} K_{r} a(\bar{b}) d V o l_{g}=-\int_{R} a\left(\overline{L_{r+1} b}\right) d V o l_{g} \\
& \Delta_{r+1} K_{r}=K_{r} \Delta_{r} \\
& \Delta_{r} L_{r+1}=L_{r+1} \Delta_{r+1} \\
& \text { whenever } g \text { has constant curvature }-1 \text {. }
\end{aligned}
$$

See e.g. [16], [17]. Recall that a Beltrami differential $\mu$ is harmonic if and only if we can write $\mu=\frac{\bar{q}}{d V o_{g}}$, where $q$ is a holomorphic quadratic differential, and this is the case if and only if $K_{-2}(\mu)=0$.

We say that a metric $h \in \mathcal{M}$ is critical with respect to the fixed metric $\mathrm{g}$ if the identity map is critical for $T_{\partial}$ between $(R, g)$ and $(R, h)$.

By the above computation we conclude

Theorem 3.2. The metric $h$ is critical with respect to $g$ if and only if

$$
K_{-2}\left(Q_{h}\right)=L_{0}\left(P_{h}\right)
$$

Let us denote with $\mathcal{C}_{g}$ the space of metrics which are critical with respect to $g$.

Remark 2. If $\phi$ is an orientation preserving diffeomorphism of $R$, then

$$
T_{\partial}(\phi, g, h)=T_{\partial}\left(I, g, \phi^{*}(h)\right)=\int_{R}\left(P_{\phi^{*}(h)}+1\right) d V o l_{g}
$$

When the metrics have negative curvature, we can interpret the critical point of $T_{\partial}$ as area minimizing maps in suitable Riemannian 4-manifolds. Let $g$ and $h$ be two metrics on $R$, both with strictly negative curvature, consider the product $R \times R$ with the metric

$$
s(x, y)=\sqrt{\frac{K_{g}(x)}{K_{h}(y)}} g(x)+\sqrt{\frac{K_{h}(y)}{K_{g}(x)}} h(y) .
$$


The above Riemannian manifold will henceforth be denoted by $R * R$. We have the following

Lemma 3.3. Let $g$ and $h$ be two metrics with strictly negative curvature, let $\phi: R \rightarrow R$ be a non antiholomorphic smooth map, and $\tilde{\phi}: R \rightarrow R * R$ be the graph map given by $\tilde{\phi}(x)=(x, \phi(x))$. Then $\phi$ is critical with respect to $T_{\partial}(g, h)$, if and only if $\tilde{\phi}$ is minimal and with lagrangian image with respect to the simplectic form $K_{g} d V o_{g}-K_{h} d V o l_{h}$. In this case $\operatorname{Vol}(\tilde{\phi}(R))=$ $2 T(g, h, \phi)$.

Proof. Set $p=\tilde{\phi}^{*}(s), a=\sqrt{-K_{g}}, b=\sqrt{-K_{h}}$. We have $p=\frac{a}{b(\phi)} g+\frac{b(\phi)}{a} \phi^{*}(h)$. If we write $g=\rho^{2} d z d \bar{z}, h=\sigma^{2} d w d \bar{w}, p=\delta^{2} d \zeta d \bar{\zeta}$ and

$$
\phi^{*}(h)=\rho^{2}\left((P+1) Q d \bar{z}^{2}+\left((P+1)^{2}+|Q|^{2}\right) d z d \bar{z}+(P+1) \bar{Q} d z^{2}\right),
$$

then

$$
\begin{aligned}
& \left.\left(\frac{d V o l_{p}}{d V o l_{g}}\right)^{2}=\left(\left(\frac{a}{b(\phi)}+\frac{b(\phi)}{a}\left((P+1)^{2}+|Q|^{2}\right)\right)\right)^{2}-4\left|\frac{b(\phi)}{a}(P+1) Q\right|^{2}\right) \\
& =\left(\frac{a^{2}}{b^{2}(\phi)}+\frac{b^{2}(\phi)}{a^{2}}\left((P+1)^{2}+|Q|^{2}\right)^{2}+2\left((P+1)^{2}+|Q|^{2}\right)\right) \\
& \left.\quad-4\left|\frac{b(\phi)}{a}(P+1) Q\right|^{2}\right) \\
& =\left(\frac{a^{2}}{b^{2}(\phi)}+\frac{b^{2}(\phi)}{a^{2}}\left((P+1)^{2}-|Q|^{2}\right)+4(P+1)^{2}-2\left((P+1)^{2}-|Q|^{2}\right)\right) \\
& =4(P+1)^{2}+\left(\frac{a}{b(\phi)}-\frac{b(\phi)}{a} \frac{d V o l_{\phi^{*}(h)}}{d V o l_{g}}\right)^{2} .
\end{aligned}
$$

Set $q(\phi)=\left(\frac{a}{b(\phi)}-\frac{b(\phi)}{a} \frac{d V o l_{\phi^{*}(h)}}{d V o l_{g}}\right)$. Note that the map $\phi$ preserves curvature if and only if $\tilde{\phi}$ has lagrangian image if and only if $q(\phi)=0$. Let $\tilde{\phi}_{t}$ be a variation of $\tilde{\phi}$ which for small t may be assumed to be given by the graphs of a variation $\phi_{t}$ of $\phi$. Then

$$
\frac{\partial\left(\operatorname{Area}\left(\tilde{\phi}_{t}(R)\right)\right.}{\partial t}{ }_{\mid t=0}=\int_{R} \frac{4(P+1) \frac{\partial P}{\partial t}\left|t=0+q(\phi) \frac{\partial q\left(\phi_{t}\right)}{\partial t}\right| t=0}{\sqrt{4(P+1)^{2}+q(\phi)^{2}}} d V o l_{g} .
$$

Now the lemma follows from Lemma 2.2 and Remark 2. 
Remark 3. Let $\phi$ be a critical and $\Psi=\left(\Psi_{1}, \Psi_{2}\right)$ be its graph map, then we define

$$
\begin{aligned}
& \Phi_{1}(\Psi)=-K_{g}\left(\Psi_{1}\right) \rho^{2} \partial_{z} \Psi_{1} \partial_{z} \bar{\Psi}_{1} \\
& \Phi_{2}(\Psi)=-K_{h}\left(\Psi_{2}\right) \sigma^{2} \partial \Psi_{2} \partial_{w} \bar{\Psi}_{2} \\
& A_{1}(\Psi)=\log \left(\frac{K_{h}\left(\Psi_{2}\right)}{K_{g}\left(\Psi_{1}\right)}\right) \\
& A_{2}(\Psi)=\log \left(\frac{K_{g}\left(\Psi_{1}\right)}{K_{h}\left(\Psi_{2}\right)}\right) \\
& \mathcal{H}_{1}(\Psi)=-K_{g}\left(\Psi_{1}\right) \frac{\rho^{2}\left|\partial \Psi_{1}\right|^{2}}{\delta^{2}} \\
& \mathcal{H}_{2}(\Psi)=-K_{h}\left(\Psi_{2}\right) \frac{\sigma^{2}\left|\partial \Psi_{2}\right|^{2}}{\delta^{2}} \\
& \mathcal{L}_{1}(\Psi)=-K_{g}\left(\Psi_{1}\right) \frac{\rho^{2} \mid \partial^{2} \Psi^{2}}{\delta^{2}} \\
& \mathcal{L}_{2}(\Psi)=-K_{h}\left(\Psi_{2}\right) \frac{\sigma^{2}\left|\partial \Psi_{2}\right|^{2}}{\delta^{2}} .
\end{aligned}
$$

Let us collect some formulas for future reference. Since $\Phi$ is harmonic, conformal, and with lagrangian image, it satisfies the following equations (in the $\zeta$ coordinates.)

$$
\left\{\begin{array}{l}
\bar{\partial} \partial \Psi_{1}+\partial_{z}\left(\log \rho^{2}\right) \bar{\partial} \Psi_{1} \partial \Psi_{1}=1 / 2\left(\bar{\partial} \Psi_{1} \partial_{\xi} A_{1}+\partial \Psi_{1} \bar{\partial}_{\xi} A_{1}\right) \\
\bar{\partial} \partial \Psi_{2}+\partial_{w}\left(\log \sigma^{2}\right) \bar{\partial} \Psi_{2} \partial \Psi_{2}=1 / 2\left(\bar{\partial} \Psi_{2} \partial_{\xi} A_{2}+\partial \Psi_{2} \bar{\partial}_{\xi} A_{2}\right)
\end{array}\right.
$$

Moreover from above we derive the Bochner type formula

$$
\begin{gathered}
\partial_{\zeta} \bar{\partial}_{\zeta}\left(\log \left(\frac{\left|\partial \Psi_{1}\right|^{2}}{\left|\bar{\partial} \Psi_{1}\right|^{2}}\right)=\right. \\
2 \partial_{z} \bar{\partial}_{z}\left(\log \left(\rho^{2}\right)\left(\left|\partial \Psi_{1}\right|^{2}-\left|\bar{\partial} \Psi_{1}\right|^{2}\right)\right. \\
\left.-(1 / 2) \partial\left(\frac{\left(\left|\partial \Psi_{1}\right|^{2}-\left|\bar{\partial} \Psi_{1}\right|^{2}\right) \partial_{\zeta} A_{1}}{\partial \Psi_{1} \partial \bar{\Psi}_{1}}\right)\right) \\
-(1 / 2) \bar{\partial}\left(\frac{\left(\left|\partial \Psi_{1}\right|^{2}-\left|\bar{\partial} \Psi_{1}\right|^{2}\right) \bar{\partial}_{\zeta} A_{1}}{\bar{\partial} \Psi_{1} \bar{\partial} \bar{\Psi}_{1}}\right)
\end{gathered}
$$

(see Appendix)

Remark 4. If both metric $g$ and $h$ have constant negative curvature, it is observed in [12] that every minimal immersion has lagrangian image.

\section{The constant curvature case.}

Throughout this section we will assume that both $g$ and $h$ have constant Gauss curvature -1 . 
Lemma 4.1. There is a unique critical point of $T_{\partial}$ in the homotopy class of an orientation preserving diffeomorphism.

Proof. Let $\phi_{0}$ an orientation preserving diffeomorphism, let $\tilde{\phi}_{0}$ be its graph map; It follows from Schoen-Yau [11], that there exists a metric $m\left(g, h, \phi_{0}\right)$ of constant curvature -1 on $R$, and a map $\Psi=\left(\Psi_{1}, \Psi_{2}\right):\left(R, m\left(g, h, \phi_{0}\right)\right) \rightarrow$ $R * R$ which is harmonic, conformal, and homotopic to $\tilde{\phi}_{0}$. Since $K_{g}=K_{h} \equiv$ -1 , then $R * R=(R, g) \times(R, h)$ and both $\Psi_{1}, \Psi_{2}$ are harmonic. Then, by [10], they are orientation preserving diffeomorphism, and by Remark 4, the map $\Psi_{2} \circ \Psi_{1}{ }^{-1}$ is a critical point for $T_{\partial}$. It follows from Schoen [12], that there exists only one such critical point. Therefore also the metric $m\left(g, h, \phi_{0}\right)$ is uniquely determined by $g h$ and $\phi_{0}$.

Remark 5. Note that, in the case of constant curvature, the lagrangian condition implies that the maps $\Psi_{1}$ and $\Psi_{2}$ have the same energy density, so they have the same energy. Moreover we have

$$
2 T_{\partial}=\operatorname{Vol}(\tilde{\phi})=E\left(\Psi_{1}\right)+E\left(\Psi_{2}\right)=2 E\left(\Psi_{1}\right)=2 E\left(\Psi_{2}\right) .
$$

Theorem 4.2. Let $g$ and $h$ be hermitian metrics on $R$, both with constant curvature -1 , then

(i) Suppose an homotopy class of maps of $R$ into itself contains an antiholomorphic non constant map, then there is only one such map and it is the unique critical point of $T_{\partial}$ in the class.

(ii) Suppose an homotopy class contains the constants, then these are the only critical points of $T_{\partial}$ in the class.

(iii) Suppose an homotopy class contains an orientation preserving diffeomorphism, then there exists a unique critical point of $T_{\partial}$ in the class. Such point is a simplectic diffeomorphism.

(iv) In any other homotopy class there is no critical points of $T_{\partial}$.

Proof. Two non constant homotopic antiholomorphic maps coincide, an antiholomorphic map homotopic to a constant is itself constant. The Theorem then follows from Remark 1 and Lemma 4.1 
If the starting diffeomorphism $\phi_{0}$ is chosen to be the identity, and if for fixed $g$ we set $m_{g}(h)$ to be the Teichmüller class of $m(g, h, I)$, then $m_{g}(h)$ defines a smooth map from $\mathcal{T}$ into $\mathcal{T}$. Where $\mathcal{T}=\mathcal{M}_{-1} / \mathcal{D}_{0}$ is Teichmüller space.

Given two metrics $g$ and $h$ with constant curvature -1 , let $\Phi(g, h)$ be the $(2,0)$ part of $\mathrm{h}$ with respect to $\mathrm{g}$, and let $E(g, h)$ be the energy of the harmonic map from $(R, g)$ to $(R, h)$ homotopic to the identity.

Then the map $m_{g}$ described above is defined by the following equation

$$
\left.\Phi\left(m_{g}(h), g\right)\right)+\Phi\left(m_{g}(h), h\right)=0 .
$$

Also, if $\phi$ denotes the corresponding critical point of $T_{\partial}$, then, by Remark 5 we have

$$
T_{\partial}(\phi, g, h)=E\left(m_{g}(h), g\right)=E\left(m_{g}(h), h\right) .
$$

Equation (4.1), implies that the Teichmüller class of $g$ is the only fixed point of $m_{g}$. Moreover, since, according to Wolf, [14], the holomorphic quadratic differentials give coordinates in Teichmüller space, it follows that $m_{g}$ is bijective, and by the implicit function Theorem, $m_{g}$ is a diffeomorphism of Teichmüller space. Again from equation (4.1) we derive that $m_{g}(h)=m_{h}(g)$.

Lemma 4.3. We have that the differential of $m_{g}$ at $g$ equals $1 / 2$ Identity.

Proof. Consider the energy of harmonic maps as a function $E: \mathcal{T} \times \mathcal{T} \rightarrow \mathbf{R}$. Recall that $-\Phi(h, g)$ is the Weil Peterson gradient of the energy with respect to the first variable, see [13], hence equation (4.1) says

$$
\frac{\partial E}{\partial x_{i}}\left(m_{g}(h), g\right)=-\frac{\partial E}{\partial x_{i}}\left(m_{g}(h), h\right)
$$

Differentiating with respect to $\mathrm{h}$ and evaluating in $\mathrm{g}$ we find

$$
2 \sum_{j} \frac{\partial^{2} E}{\partial x_{i} \partial x_{j}}(g, g) \frac{\partial m_{g}^{j}}{\partial x_{k}}=-\frac{\partial^{2} E}{\partial x_{i} \partial y_{k}}(g, g) \text {. }
$$

Since $\mathrm{E}$ takes its minimum on the diagonal of $\mathcal{T} \times \mathcal{T}$, by differentiating the equality $\frac{\partial E}{\partial x_{i}}(h, h)=0$, and evaluating at $g$, we find $\frac{\partial^{2} E}{\partial x_{i} \partial x_{j}}(g, g)+$ 
$\frac{\partial^{2} E}{\partial x_{i} \partial y_{j}}(g, g)=0$. Since the Hessian of $E(\cdot, g)$ at $g$ is invertible, the Lemma follows.

We will describe later a metric on the space $\mathcal{M}$ of metrics on $\mathrm{R}$, such that $m_{g}(h)$ is the Teichmüller class of the medium point of a geodesic joining suitable representatives of $g$ and $h$. Let us define $T_{\partial}[g]: \mathcal{T} \rightarrow \mathbf{R}$ by $T_{\partial}[g](h)=T_{\partial}(\phi, g, h)$ where $\phi$ is the unique critical point of $T_{\partial}$ homotopic to the identity.

Theorem 4.4. The function $T_{\partial}[g]$ is smooth and proper, its absolute minimum is taken at $g$, which is also its only critical point. Moreover the hessian of $T_{\partial}[g]$ at $g$ is two times the the Weil-Petersson metric.

Proof. The Theorem follows from the analogous results for the energy function, see [13], together with formula (4.2) and Lemma 4.3.

Remark 6. It is easy to see from the above, that in the case of the sphere with constant curvature +1 , the only critical points of $T_{\partial}$ are the antiholomorphic maps and the isometries. In the case of the flat torus $T$ the situation is more complicated, in fact, for example, the map $z \rightarrow z+\bar{z}$, from the complex plane into itself, induces a critical point $\phi$ of $T_{\partial}$, such that $\partial \phi$ is nowhere zero, however $\phi$ is not an isomorphism.

\section{The generalized Lorentzian metric.}

Let $g \in \mathcal{M}$, given by $g=\rho^{2} d z d \bar{z}$. Let $k$ be a tangent vectors to $\mathcal{M}$ at $\mathrm{g}$, so $k$ is a bilinear symmetric form. Let $\tilde{k}$ be the contraction of $k$ with the metric $g$. Define the quadratic form on $T_{g}(\mathcal{M})$ given by

$$
\|k\|_{g}^{2}=-1 / 4 \int_{R} \operatorname{det}(\tilde{k}) d V o l_{g} .
$$

If $k_{1}={\overline{A^{\prime}}}_{1} \rho^{2} d z d \bar{z}+2 B^{\prime}{ }_{1} \rho^{2} d z d \bar{z}++A^{\prime}{ }_{1} \rho^{2} d z d \bar{z}$ and $k_{2}={\overline{A^{\prime}}}_{2} \rho^{2} d z d \bar{z}+$ $2 B^{\prime}{ }_{2} \rho^{2} d z d \bar{z}++A^{\prime}{ }_{2} \rho^{2} d z d \bar{z}$ then the Lorentzian metric resulting from polarization is

$$
<<k_{1}, k_{2}>>_{g}=\int_{R}\left(\overline{A^{\prime}}{ }_{1} A_{2}^{\prime} / 2+{\overline{A^{\prime}}}_{2} A^{\prime}{ }_{1} / 2-B_{1}^{\prime}{ }_{1}^{\prime}{ }_{2}\right) d V o l_{g} .
$$

The above metric is "generalized Lorentzian" since the spaces $\left\{P^{\prime}=0\right\}$ and $\left\{Q^{\prime}=0\right\}$ decompose the tangent space to $\mathcal{M}$ at $g$ in orthogonal direct 
sum; moreover the metric is positive definite on the space $\left\{P^{\prime}=0\right\}$ locally parameterized by a pair of real functions, whereas it is negative definite on $\left\{Q^{\prime}=0\right\}$, which is locally parameterized by a single real function. Note that the group $\mathcal{D}$ of orientation preserving diffeomorphisms of $R$ acts by pull-back on $\mathcal{M}$. Then we have the following

Lemma 5.1. The above scalar product is $\mathcal{D}$ invariant.

Proof. It is a straightforward calculation from the definitions.

Proposition 5.2. The orthogonal space with respect to $\left\langle<>>\right.$ to the $\mathcal{D}_{0}$ orbit is the tangent space to $\mathcal{C}_{g}$.

Proof. Let $\phi_{t}:(-\varepsilon, \varepsilon) \rightarrow \mathcal{D}$, be a one parameter group of diffeomorphisms with $\phi_{0}=I$ and $\phi_{0}^{\prime}=u$. Then the Lie derivative $\mathcal{L}_{u}(g)$ is given by

$$
\mathcal{L}_{u}(g)=\frac{\partial}{\partial t}\left(\phi_{t}^{*}(g)\right)_{t=0}=\rho^{2}(\partial \bar{u}) d z^{2}+\left(\partial\left(\rho^{2} u\right)+\bar{\partial}\left(\rho^{2} \bar{u}\right)\right) d z d \bar{z}+\rho^{2}(\bar{\partial} u) d \bar{z}^{2} .
$$

If we set $\theta=\left(\rho^{2} d z d \bar{z}\right)^{1 / 2} u$, then $\theta$ is in $S(-1)$ and

$$
\mathcal{L}_{u}(g)=\left(L_{-1}(\theta)+2 \operatorname{Re}\left(K_{-1}(\theta)\right)+\overline{L_{-1}(\theta)}\right) g .
$$

In other words a vector $\left(\begin{array}{c}P^{\prime} \\ Q^{\prime}\end{array}\right)$ is tangent to the $g$ orbit, if and only if there exists an element $\theta \in S(-1)$ such that $P^{\prime}=\operatorname{Re} K_{-1}(\theta), Q^{\prime}=L_{-1}(\theta)$. If we write the metric $v$ in the form $v=\left(\bar{Q}^{\prime}+2 P^{\prime}+Q^{\prime}\right) g$ then we obtain

$$
\begin{gathered}
<<v, \mathcal{L}_{u}(g)>>=\operatorname{Re} \int_{R}\left(L_{-1}(\theta) \bar{Q}^{\prime}-K_{-1}(\theta) P^{\prime}\right) d V o l_{g} \\
=-\operatorname{Re} \int_{R}\left(\theta\left(\overline{K_{-2}\left(Q^{\prime}\right)-L_{0}\left(P^{\prime}\right)}\right) d V o l_{g} .\right.
\end{gathered}
$$

Since $\theta$ is arbitrary, the Lemma follows from Theorem 3.2.

Lemma 5.3. If $g$ is a metric with negative curvature everywhere, then the Lorentzian metric is positive definite on the $\mathcal{D}_{0}$ orbit of $g$. 
Proof. Let $\mathcal{L}_{u} g$ be a vector in $T_{g}\left(\mathcal{D}_{0} g\right)$, we have

$$
\begin{aligned}
& \left\|\mathcal{L}_{u} g\right\|^{2}=\int_{R}\left(\left|L_{-1}(\theta)\right|^{2}-\left(\operatorname{Re}\left(K_{-1}(\theta)\right)^{2}\right) d V o l_{g}\right. \\
& =\int_{R}\left(\left|L_{-1}(\theta)\right|^{2}-\left|K_{-1}(\theta)\right|^{2}+\left(\operatorname{ImK}_{-1}(\theta)\right)^{2}\right) d V o l_{g} \\
& =\int_{R}\left(\left|L_{-1}(\theta)\right|^{2}+\left(L_{0} K_{-1}(\theta) \bar{\theta}+\left(\operatorname{Im}_{-1}(\theta)\right)^{2}\right) d V o l_{g}\right. \\
& =\int_{R}\left(\left|L_{-1}(\theta)\right|^{2}+K_{-2} L_{-1}(\theta) \bar{\theta} d V o l_{g}\right. \\
& +\int_{R}\left(-\frac{K_{g}}{2}|\theta|^{2}+\left(\operatorname{Im}_{-1}(\theta)\right)^{2}\right) d V \operatorname{Vol}_{g} \\
& =\int_{R}\left(\left(\operatorname{Im}_{-1}(\theta)\right)^{2}-\frac{K_{g}}{2}|\theta|^{2}\right) d \operatorname{Vol}_{g} .
\end{aligned}
$$

Proposition 5.4. If $g$ is a metric of constant curvature -1 , then we have the equality:

$$
\begin{aligned}
\left(\left(T_{g}\left(\mathcal{D}_{0} g\right)\right)^{\perp}\right. & \left.\bigcap T_{g}\left(\mathcal{M}_{-1}\right)\right) \\
& =\left\{\left(\begin{array}{c}
0 \\
Q^{\prime}
\end{array}\right) \text { with } Q^{\prime} \text { harmonic Beltrami differential }\right\}
\end{aligned}
$$

It follows that the restriction to $\mathcal{M}_{-1} / \mathcal{D}_{0}$ of the Lorentzian metric induced on $\mathcal{M} / \mathcal{D}_{0}$ coincides with the Weil-Petersson metric. Moreover the Lorentzian metric is positive definite on $\mathcal{M}_{-1}$.

Proof. By the proof of Lemma 4.1 we see that the projection map from $\mathcal{C}_{g} \cap \mathcal{M}_{-1}$ into $\mathcal{M}_{-1} / \mathcal{D}_{0}$ is an isomorphism. In particular we have $T_{g}\left(\mathcal{M}_{-1}\right)=T_{g}\left(\mathcal{D}_{0} g\right) \oplus T_{g}\left(\mathcal{C}_{g}\right)$, which, by Proposition 5.2, coincides with $T_{g}\left(\mathcal{D}_{0} g\right) \oplus\left(\left(T_{g}\left(\mathcal{D}_{0} g\right)\right)^{\perp} \cap T_{g}\left(\mathcal{M}_{-1}\right)\right)$. Hence the space

$$
\left(\left(T_{g}\left(\mathcal{D}_{0} g\right)\right)^{\perp} \cap T_{g}\left(\mathcal{M}_{-1}\right)\right)
$$

has complex dimension $3 g e n u s(R)-3$. However, by Theorem 3.2 and by Theorem 2.4.1 of [13], it contains all the vectors of the form $\left(\begin{array}{c}0 \\ Q^{\prime}\end{array}\right)$ with $Q^{\prime}$ harmonic, and equality $\left(^{*}\right)$ follows. The Lorentzian metric is positive definite on $T_{g}\left(\mathcal{M}_{-1}\right)$ because of $\left({ }^{*}\right)$ together with Lemma 5.3 .

Theorem 5.5. If we identify the tangent space to $\mathcal{M}$ at $g$ with $S(0) \times$ $S(-2)$ via the map $d \alpha$, then the map $\Psi$ given in Proposition 3.1 becomes the exponential map of the Lorentzian metric. 
Proof. We already observed that $\Psi(0,0)=g$ and $d \Psi(0,0)=I$. Let $h$ be a metric with coordinates $P_{h}, Q_{h}$ with respect to $g$, then the square norm of a tangent vector to $h$ is given by

$$
\left\|\left(P^{\prime}, Q^{\prime}\right)\right\|^{2}=\int_{R}\left(\left|Q^{\prime}\right|^{2}-{P^{\prime}}^{2}\right)-1 / 4 \int_{R} \frac{\left(\bar{Q} Q^{\prime}-Q \bar{Q}^{\prime}\right)^{2}}{(P+1)^{2}-|Q|^{2}} d V o l_{g} .
$$

The energy of a path $\gamma(t)$ is

$$
\begin{aligned}
& E(\gamma)=\int_{0}^{1} \int_{R}\left(\left|Q^{\prime}(t)\right|^{2}-{P^{\prime}}^{2}(t)\right) d V o_{g} d t \\
& -1 / 4 \int_{0}^{1} \int_{R} \frac{\left(\overline{\left.Q(t) Q^{\prime}(t)-Q(t) Q^{\prime}(t)\right)^{2}}\right.}{(P(t)+1)^{2}-|Q(t)|^{2}} d V o l_{g} d t .
\end{aligned}
$$

Take a variation $\gamma(t, s)$ with $\gamma(0, s)=\gamma(0)$ and $\gamma(1, s)=\gamma(1)$. Let us indicate with' the differentiation with respect to $t$ and with ' the one with respect to $s$. Differentiating the energy with respect to $s$ and evaluating at $(0,0)$ we obtain

$$
\frac{\partial E(t, s)}{\partial s}=\int_{0}^{1} \int_{R}\left(Q^{\prime} \bar{Q}^{\prime}+Q^{\prime} \bar{Q} \cdot-2 P^{\prime} P^{\prime}\right) d V o l_{g} d t .
$$

(Recall that $Q(t, 0)=t Q^{\prime}$ ). Integrating by parts and observing that $P^{\prime \prime}(t, 0)=Q^{\prime \prime}(t, 0)=0$, we find that $\frac{\partial E(t, s)}{\partial s} \mid s=0=0$.

Remark 7. It follows from the above Proposition that the geodesics are the paths of the form $P(t)=t P^{\prime}, Q(t)=t Q^{\prime}$. In particular every two points in $\mathcal{M}$ can be joined by a unique geodesic. We deduce from the formula for the square norm of a vector tangent to $\mathcal{M}$, and from Remark 2 that the energy of the geodesic joining $\mathrm{g}$ and $\mathrm{h}$ is given by $E(\gamma)=2 T(I, g, h)-\operatorname{Vol}(g)-\operatorname{Vol}(h)$.

Remark 8. Let us consider the geodesics $\gamma(t), t \geq 0$ with $\gamma^{\prime}(0)=\left(\begin{array}{c}P^{\prime} \\ Q^{\prime}\end{array}\right)$.

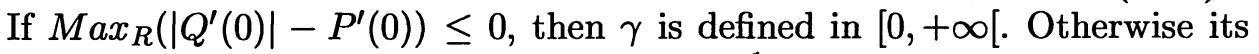
maximum interval of definition is $\left[0, \frac{1}{\max _{R}\left(\left|Q^{\prime}(0)\right|-P^{\prime}(0)\right)}[\right.$.

Theorem 5.6. Let $q: \mathcal{M} \rightarrow \mathcal{M} / \mathcal{D}_{0}$, be the projection map, let $\gamma(t)=$ $\left(\begin{array}{c}t P^{\prime} \\ t Q^{\prime}\end{array}\right), t \in[0,1]$ be a Lorentzian geodesic in $\mathcal{M}$ with $\gamma(0)=g$ and $\gamma^{\prime}(0)=$ $\left(\begin{array}{c}P^{\prime} \\ Q^{\prime}\end{array}\right)$. Then the following facts are equivalent 
(i) $\gamma^{\prime}\left(t_{0}\right)$ is orthogonal to the $\mathcal{D}_{0}$ orbit of $\gamma\left(t_{0}\right)$ for some $t_{0} \in[0,1]$

(ii) $\gamma^{\prime}(t)$ is orthogonal to the $\mathcal{D}_{0}$ orbit of $\gamma(t)$ for every $t \in[0,1]$

(iii) $\gamma\left(t_{0}\right)$ is critical with respect to $g$ for some $\left.\left.t_{0} \in\right] 0,1\right]$

(iv) $\gamma(t)$ is critical with respect to $g$ for every $t \in[0,1]$.

If the above conditions are satisfied, then $q(\gamma)$ is a geodesic in $\mathcal{M} / \mathcal{D}_{0}$ with the induced Lorentzian metric.

Proof. From Proposition 5.2 and Theorem 3.2 it follows that $\gamma^{\prime}(0)$ is orthogonal to the $\mathcal{D}_{0}$ orbit of $g$, if and only if (iii) is satisfied, if and only if (iv) is satisfied. Assume that $t_{0} \in[0,1]$ and $\gamma^{\prime}\left(t_{0}\right)$ is orthogonal to the $\mathcal{D}_{0}$ orbit of $\gamma\left(t_{0}\right)$. Let us consider the complex structure on $R$ that makes $h_{0}=\gamma\left(t_{0}\right)$ conformal. We find as above that for every $t \in[0,1]$ the metric $\gamma(t)$ is critical with respect to $\gamma\left(t_{0}\right)$, hence $\gamma\left(t_{0}\right)$ is critical with respect to $\gamma(t)$. It follows that $\gamma^{\prime}(t)$ is orthogonal to the $\mathcal{D}_{0}$ orbit of $\gamma(t)$. If $\gamma^{\prime}$ is orthogonal to the $\mathcal{D}_{0}$ orbit at every point of $\gamma$, then $q(\gamma)$ is a geodesic in $\mathcal{M} / \mathcal{D}_{0}$.

Let us now consider two metrics $g$ and $h$, both with constant curvature -1 . Let $\gamma(t)$ be the Lorentzian geodesic joining $\mathrm{g}$ and $\mathrm{h}$, assume that $h$ is critical with respect to $g$. Let $m$ be the metric corresponding to the medium point of the geodesic. Let us consider on $R$ the complex structure that makes $m$ conformal, set $m=\rho^{2} d z d \bar{z}$. Denote the corresponding exponential coordinates with $P, Q$. We must have $P_{g}=-P_{h}, Q_{g}=-Q_{h}$. We have

$$
\begin{aligned}
& d V o l_{g}=\left(\left(P_{g}+1\right)^{2}-\left|Q_{g}\right|^{2}\right) d V o l_{m} \\
& d V o_{h}=\left(\left(P_{h}+1\right)^{2}-\left|Q_{h}\right|^{2}\right) d V o l_{m}
\end{aligned}
$$

Moreover by Proposition 2.2 we have $d V o_{g}=d V o l_{h}$, hence $P_{g}=P_{h} \equiv 0$. Since $K_{-2}\left(Q_{g}\right)=L_{0}\left(P_{g}\right)$, it follows that $Q_{g}$ is harmonic, so $\Phi_{g}=\bar{Q}_{g} \rho^{2} d z d \bar{z}$ and $\Phi_{h}=-\Phi_{g}$ are holomorphic quadratic differentials. Note that $\left\|\Phi_{g}\right\|=$ $\left\|\Phi_{h}\right\|<1$. Since orientation preserving diffeomorphisms with holomorphic Hopf differential are harmonic, we conclude that the diagonal map from $(R, m) \rightarrow(R, g) \times(R, h)$ is both harmonic and conformal. In other words if $\pi: \mathcal{M} \rightarrow \mathcal{M}_{-1}$ denotes the maps that associates to every metric the unique metric, conformal to it, of constant curvature -1 , then we have $m_{g}(h)=q(\pi(m))$. Moreover

$$
K_{m} d V o l_{m}=-\left(1-|Q|^{2}\right) d V o l_{m}=-\left(1-\|\Phi\|^{2}\right) d V o l_{m}
$$


i.e.

$$
K_{m}=-\left(1-\|\Phi\|^{2}\right) .
$$

So we have the following

Proposition 5.7. There is a one-to-one correspondence

$$
(g, h) \rightarrow(m, \Phi)
$$

from the set

$$
\begin{aligned}
& X=\{(g, h): \quad g \text { and } h \text { both have constant curvature }-1, \\
& \text { and they are critical with respect to each other }\}
\end{aligned}
$$

and the set

$$
Y=\{(m, \Phi) \text {, where } m \text { is a metric }
$$

and $\Phi$ is a holomorphic quadratic differential with respect to $m$, such that $\|\Phi\|<1$, and $\left.K_{m}=-\left(1-\|\Phi\|^{2}\right)\right\}$

Here $m$ is the medium point of the Lorentzian geodesic between $g$ and $h$, whereas $\Phi$ is the $(2,0)$ part of $g$ with respect to $m$, Moreover $m_{g}(h)=$ $q(\pi(m))$.

Proof. We already described the map from $X$ to $Y$, let us describe its inverse. Set $g=\Phi+\bar{\Phi}+\left(1+\|\Phi\|^{2}\right) m$, and $h=-\Phi-\bar{\Phi}+\left(1+\|\Phi\|^{2}\right) m$, then $g$ and $h$ are critical with respect to $m$, hence by Proposition 2.2 they have constant curvature -1 , moreover the diagonal map from $(R, m)$ into $(R, g) \times$ $(R, h)$ is harmonic and conformal, in particular $g$ and $h$ are critical with respect to each other. Let $m^{\prime}$ be the medium point of the geodesics between $g$ and $h$. From above we see that the diagonal map between $\left(R, m^{\prime}\right)$ and $(R, g) \times(R, h)$ is also harmonic and conformal. It follows that $m$ and $m^{\prime}$ are conformal. Moreover the $P$ coordinates of $g$ with respect to $m$ and $m^{\prime}$ are both identically zero, so $m=m^{\prime}$. Moreover, by construction, the quadratic differential $\Phi$ is the $(2,0)$ part of $g$ with respect to $m=m^{\prime}$. The rest of the Proposition has already been proved.

Remark 9. It can by proved as above that given $(m, \Phi) \in Y$ and $\theta \in[0,2 \pi]$ the metric $g_{\theta}=e^{i \theta} \Phi+e^{-i \theta} \bar{\Phi}+\left(1+|\Phi|^{2}\right) m$, has constant curvature -1 and it is critical with respect to $m$ 
The set $Y$ was already considered by Hitchin [6], (in fact, among other things, he proved the above Remark). The system of equations

$$
\left\{\begin{array}{l}
K_{m}=-\left(1-\|\Phi\|^{2}\right) \\
\bar{\partial} \Phi=0
\end{array}\right.
$$

is the abelian vortex equation.

\section{The orthogonal space to $\mathcal{M}_{-1}$.}

Let $g$ be a metric with constant curvature -1. It follows from Proposition 5.2, Theorem 3.2 and Corollary 5.4 that a vector $\left(\begin{array}{c}P^{\prime} \\ Q^{\prime}\end{array}\right) \in T_{g}(\mathcal{M})$ is in $T_{g}\left(\mathcal{M}_{-1}\right)^{\perp}$ if and only if $Q^{\prime}$ is $L^{2}$ orthogonal to the space of harmonic Beltrami differentials and $K_{-2}\left(Q^{\prime}\right)=L_{0}\left(P^{\prime}\right)$.

Set $D=(-1 / 2(\Delta-2 I))^{-1}$, where $\Delta$ is the Laplace-Beltrami operator on $R$ with negative spectrum. The operator $D$ is known to be compact, $L^{2}$ self adjoint and positive, see for example [15]. As in [15] we can prove the following

Proposition 5.8. A vector $\left(\begin{array}{c}P^{\prime} \\ Q^{\prime}\end{array}\right) \in T_{g}(\mathcal{M})$ is orthogonal to $\mathcal{M}_{-1}$ if and only if

$$
Q^{\prime}=-2 L_{-1} L_{0} D\left(P^{\prime}\right) \text {. }
$$

Proof. Assume that $Q^{\prime}=-2 L_{-1} L_{0} D\left(P^{\prime}\right)$, and let $\mu$ be an harmonic Beltrami differential, then $\int_{R} Q^{\prime} \bar{\mu} d V o l_{g}=2 \int_{R} L_{0}\left(D\left(P^{\prime}\right) \overline{K_{-2}(\mu)} d V o l_{g}=0\right.$. On the other hand we have

$$
\begin{aligned}
4 L_{-1} K_{-2}\left(-2 L_{-1} L_{0}\left(D\left(P^{\prime}\right)\right)\right. & =\left(\Delta_{-2}-2 I\right)\left(-2 L_{-1} L_{0}\left(D\left(P^{\prime}\right)\right)\right. \\
& =-2 \Delta_{-2} L_{-1} L_{0} D\left(P^{\prime}\right)+4 L_{-1} L_{0} D\left(P^{\prime}\right) \\
& =-2\left(L_{-1} \Delta_{-1} L_{0} D\left(P^{\prime}\right)+4 L_{-1} L_{0} D\left(P^{\prime}\right)\right. \\
& =-2 L_{-1} L_{0} \Delta\left(D\left(P^{\prime}\right)\right)+4 L_{-1} L_{0} D\left(P^{\prime}\right) \\
& =4 L_{-1} L_{0}\left(P^{\prime}\right)
\end{aligned}
$$

i.e. $4 L_{-1} K_{-2}\left(Q^{\prime}\right)=4 L_{-1} L_{0}\left(P^{\prime}\right)$. However, since $R$ does not have non trivial holomorphic vector fields, it follows that $L_{-1}$ has trivial kernel. Viceversa, if $\left(\begin{array}{c}P^{\prime} \\ Q^{\prime}\end{array}\right)$ is orthogonal to $\mathcal{M}_{-1}$, then the differentials $Q^{\prime}$ and 
$-2 L_{-1} L_{0}\left(D\left(P^{\prime}\right)\right)$ have the same $K_{-2}$ image and they are both $L^{2}$ orthogonal to the harmonic differentials, hence they coincide.

Set $H=-2 L_{-1} L_{0} D$.

Proposition 5.9. We have

$$
T_{g}(\mathcal{M})=T_{g}\left(\mathcal{M}_{-1}\right) \oplus T_{g}\left(\mathcal{M}_{-1}\right)^{\perp} .
$$

Moreover the Lorentzian metric is positive definite on $T_{g}\left(\mathcal{M}_{-1}\right)$ and negative definite on its orthogonal space.

Proof. We already know that the Lorentzian metric is positive definite on $\mathcal{M}_{-1}$, in particular $\mathcal{M}_{-1}$ has trivial intersection with its orthogonal. Let $\left(\begin{array}{c}P^{\prime} \\ Q^{\prime}\end{array}\right) \in T_{g}(\mathcal{M})$, and consider the decomposition $\left(\begin{array}{c}P^{\prime} \\ Q^{\prime}\end{array}\right)=\left(\begin{array}{c}X \\ Y\end{array}\right)+$ $\left(\begin{array}{c}Z \\ W\end{array}\right)$, where $\left(\begin{array}{c}X \\ Y\end{array}\right) \in T_{g}\left(\mathcal{D}_{0} g\right)$ and $\left(\begin{array}{c}Z \\ W\end{array}\right) \in\left(T_{g}\left(\mathcal{D}_{0} g\right)\right)^{\perp}$. Decompose farther $\left(\begin{array}{c}Z \\ W\end{array}\right)$ as $\left(\begin{array}{c}Z \\ W\end{array}\right)=\left(\begin{array}{c}Z \\ Q_{1}\end{array}\right)+\left(\begin{array}{c}0 \\ Q_{2}\end{array}\right)$, where $Q_{2}$ is harmonic and $Q_{1}$ is $L^{2}$ orthogonal to the harmonic differentials. So $\left(\begin{array}{c}X \\ Y+Q_{2}\end{array}\right)$ is tangent to $\mathcal{M}_{-1}$ and $\left(\begin{array}{c}Z \\ Q_{1}\end{array}\right)$ is orthogonal to it. By Proposition 5.8, together with the properties of the complex covariant differentials $K_{r}, L_{r}$ it follows, as in [15] pag. 135, that if $\left(\begin{array}{c}P^{\prime} \\ Q^{\prime}\end{array}\right) \in\left(T_{g}\left(\mathcal{M}_{-1}\right)\right)^{\perp}$ then

$$
\int_{R}\left|Q^{\prime}\right|^{2} d V o l_{g}=\int_{R}\left|P^{\prime}\right|^{2} d V o l_{g}-\int_{R} D\left(P^{\prime}\right) P^{\prime} d V o l_{g}
$$

hence $<<\left(\begin{array}{c}P^{\prime} \\ Q^{\prime}\end{array}\right),\left(\begin{array}{c}P^{\prime} \\ Q^{\prime}\end{array}\right)>>=-\int_{R} D\left(P^{\prime}\right) P^{\prime} d V o l_{g}$. Since $D$ is a positive operator and $Q^{\prime}=H\left(P^{\prime}\right)$, we conclude that $\langle<>>$ is negative definite on $T_{g}\left(\mathcal{M}_{-1}\right)^{\perp}$.

From the above Proposition we see that the orthogonal projection from $T_{g}(\mathcal{M})$ to $T_{g}\left(\mathcal{M}_{-1}\right)$ is well defined and it is easy to show that the LeviCivita connection on $\mathcal{M}_{-1}$ coincides with the orthogonal projection of the connection on $\mathcal{M}$. 


\section{The Weil-Petersson geodesics.}

Let $g \in \mathcal{M}_{-1}$ and $\gamma(t)$ for $t \in[0,1]$ a Weil-Petersson geodesics in Teichmüller space $\mathcal{T}=\mathcal{M}_{-1} / \mathcal{D}_{0}$, with $\gamma(0)=q(g)$. Let $\tilde{\gamma}=\left(\begin{array}{c}P(t) \\ Q(t)\end{array}\right) \in \mathcal{M}_{-1}$ be the lift of $\gamma$ starting at $g$ which is, at every point, Lorentz orthogonal to the $\mathcal{D}_{0}$ orbit. So $\tilde{\gamma}$ is a Lorentzian geodesics. Note that, since the Lorentzian metric and the usual $L^{2}$ metric on $\mathcal{M}_{-1}$, (see e.g. [13]) have the same orthogonal space to the $\mathcal{D}_{0}$ orbits, then $\tilde{\gamma}$ can also be regarded as the $L^{2}$ lift of $\gamma$.

Lemma 5.10. We have $d V o l_{\gamma(t)}=d V o l_{g}$ for every $t \in[0,1]$.

Proof. Given $t_{0} \in[0,1]$ let $h_{0}=\gamma\left(\tilde{t}_{0}\right)$ and let $P_{h_{0}}, Q_{h_{0}}$ be the exponential coordinates with respect to $h_{0}$. For positive $t$ close to 0 we have

$$
\begin{aligned}
d V o l_{\gamma\left(t+t_{0}\right)}=\left(\left(\left(P\left(t+t_{0}\right)+1\right)^{2}-\right.\right. & \left.\left|Q\left(t+t_{0}\right)\right|^{2}\right) d V o l_{g} \\
& =\left(\left(P_{h_{0}}(t)+1\right)^{2}-\left|Q_{h_{0}}(t)\right|^{2} \mid\right) d V o l_{h_{0}} .
\end{aligned}
$$

Since the tangent vector to the curve at $h_{0}$ is tangent to $\mathcal{M}_{-1}$ and orthogonal to the $\mathcal{D}_{0}$ orbit, it follows from Proposition 5.4 that

$$
{\frac{\partial\left(\left(P_{h_{0}}(t)+1\right)^{2}-\left|Q_{h_{0}}(t)\right|^{2} \mid\right)}{\partial t}}_{\mid t=0}=2{\left.\frac{\partial P_{h_{0}}}{\partial t}\right|_{\mid t=0}} \equiv 0 .
$$

So

$$
\frac{\partial\left((P+1)(t)^{2}-|Q(t)|^{2} \mid\right)}{\partial t}{ }_{\mid t=t_{0}} \equiv 0
$$

Hence

$$
(P+1)^{2}-|Q|^{2} \equiv 1
$$

Lemma 5.11. We have the equation $\frac{\partial^{2} Q}{\partial t^{2}}(0)=H\left(\left|\frac{\partial Q}{\partial t}(0)\right|^{2}\right)$.

Proof. Since $\left(\begin{array}{c}P \\ Q\end{array}\right)$ gives the exponential coordinates for the Lorentzian metric and $\tilde{\gamma}$ is a geodesic in $\mathcal{M}_{-1}$, then the vector $\left(\begin{array}{c}P^{\prime \prime}(0) \\ Q^{\prime \prime}(0)\end{array}\right)$ is orthogonal 
to $\mathcal{M}_{-1}$. Moreover by differentiating twice equation (5.1) and evaluating at 0 we find $P^{\prime \prime}(0)=\left|Q^{\prime}(0)\right|^{2}$. Hence the Lemma follows from Proposition 5.8

Let $g$ be a metric let $z$ be local holomorphic coordinates such that $g=$ $\rho^{2} d z d \bar{z}$. Let $h_{0}$ be another metric with coordinates $P^{h_{0}}, Q^{h_{0}}$ with respect to $g$. Let us consider on $R$ holomorphic local coordinates such that $h_{0}=$ $\sigma^{2} d w d \bar{w}$. Let $f=w^{-1} z$. So we have $f^{*}\left(\sigma^{2} d w d \bar{w}\right)$ is conformal to $h_{0}$, hence

$$
\begin{gathered}
\frac{\rho^{2} f \frac{\overline{\partial f}}{\partial z} \frac{\partial f}{\partial \bar{z}} d \bar{z}}{\sigma^{2} d z}=r(P+1) Q \\
\frac{\rho^{2} f\left|\frac{\partial f}{\partial z}\right|^{2}}{\sigma^{2}}=r(P+1)^{2} .
\end{gathered}
$$

for some positive smooth function $r$. It follows that

$$
\frac{\partial f}{\partial \bar{z}}=\mu \frac{\partial f}{\partial z}
$$

where

$$
\mu=\frac{Q_{h_{0}}}{P_{h_{0}}+1}
$$

Since $h_{0}$ is a metric, then $|\mu|<1$ and the Beltrami equation (5.2) admits a global solution $f^{\mu}$ which is a diffeomorphism of $R$. Since any pair of local solution of (5.2) differ by a holomorphic map, see [1], it follows that $f^{\mu}$ : $(R, g) \rightarrow(R, h)$ is holomorphic, by changing the holomorphic coordinates, if needed, we might assume that $w=f^{\mu}(z)$, hence that the function $r$ is identically 1 , i. e.

$$
\begin{gathered}
\frac{\rho^{2} f^{\mu} \frac{\overline{\frac{\partial f^{\mu}}{\partial z}}}{\partial f^{\mu}} \frac{\partial \bar{z}}{\partial \bar{z}}}{\sigma^{2} d z}=(P+1) Q \\
\frac{\rho^{2} f^{\mu}\left|\frac{\partial f^{\mu}}{\partial z}\right|^{2}}{\sigma^{2}}=(P+1)^{2} .
\end{gathered}
$$

We are going to compute the geodesics equation by pulling back equation in Lemma 5.11 on a given point $t_{0}$ of $\tilde{\gamma}$, via a global solution $f^{\mu}$ of the Beltrami equation. So let us fix $t_{0} \in[0,1]$ and $t$ positive close to 0 . Let $z$ be holomorphic local coordinates for the complex structure induced by $g$ and $w$ holomorphic local coordinates for the one induced by $h_{0}$, with these notations we have

$$
\left(P\left(t+t_{0}\right)+1\right) \bar{Q}\left(t+t_{0}\right) \rho^{2} d z d \bar{z}
$$




$$
\begin{gathered}
+\left(\left(P\left(t+t_{0}\right)+1\right)^{2}+\left|Q\left(t+t_{0}\right)\right|^{2}\right) \rho^{2} d z d \bar{z} \\
+\left(P\left(t+t_{0}\right)+1\right) Q\left(t+t_{0}\right) \rho^{2} d z d \bar{z} \\
=f^{\mu *}\left(\left(P_{h_{0}}(t)+1\right) \overline{Q_{h_{0}}(t)} \sigma^{2} d w d \bar{w}\right) \\
+f^{\mu *}\left(\left(\left(P_{h_{0}}(t)+1\right)^{2}+\left.\left|Q_{h_{0}}\right|(t)\right|^{2}\right) \sigma^{2} d w d \bar{w}\right) \\
+f^{\mu *}\left(\left(P_{h_{0}}(t)+1\right) Q_{h_{0}}(t) \sigma^{2} d w d \bar{w}\right) .
\end{gathered}
$$

If $B=\tilde{B}(w) \frac{d \bar{w}}{d w}$ is a Beltrami differential, then $f^{\mu *}(B)$ is given, in holomorphic local coordinates $z$, by the equation

$$
f^{\mu *}(B)=\tilde{B}\left(f^{\mu}\right) \frac{\overline{\frac{\partial f^{\mu}}{\partial z}}}{\frac{\partial f^{\mu}}{\partial z}} \frac{d \bar{z}}{d z} .
$$

and for every smooth test function $\theta$ on $\mathrm{R}$, we have, by definition (Here $H_{h_{0}}$ is the operator $H$ computed with respect to the metric $h_{0}$.)

$$
\left(f^{\mu *}\left(H_{h_{0}}\right)\right)\left(\theta\left(f^{\mu}\right)\right)=f^{\mu *}\left(H_{h_{0}}(\theta)\right)
$$

Moreover observe that $\mu_{h_{0}}{ }^{\prime}(0)=Q_{h_{0}}{ }^{\prime}(0)$. From general Teichmüller theory (see for example [16]), we know that

$$
f^{\mu *}\left(Q_{h_{0}}{ }^{\prime}(0)\right)=\frac{\partial \mu}{\partial t}\left(1-|\mu|^{2}\right)^{-1} .
$$

If we keep in mind the equations (5.2), (5.3) and equation (5.1) relative to the exponential coordinates based on $h_{0}$, then by differentiating equation (5.4) twice, evaluating at 0 taking the $(0,2)$ and $(1,1)$ part divided by $\rho^{2} d z d \bar{z}$, we find the equations

$$
\begin{gathered}
{\frac{\partial^{2}((P+1) Q)}{\partial t^{2}}}_{\mid t=t_{0}}=f^{\mu *}\left(H_{h_{0}}\right)\left(\left|Q_{h_{0}}{ }^{\prime}(0)\right|^{2}\left(f^{\mu}\right)\right)(P+1)^{2} \\
+4\left|Q_{h_{0}}{ }^{\prime}(0)\right|^{2}\left(f^{\mu}\right)(P+1) Q+\left(\overline{f^{\mu *}\left(H_{h_{0}}\right)}\left(\left|Q_{h_{0}}{ }^{\prime}(0)\right|\left(f^{\mu}\right) Q\right) Q .\right.
\end{gathered}
$$

and

$$
\begin{gathered}
{\frac{\partial^{2}\left((P+1)^{2}+|Q|^{2}\right)}{\partial t^{2}}}_{\mid t=t_{0}}=2\left(f^{\mu *}\left(H_{h_{0}}\right)\right)\left(\left|Q_{h_{0}}{ }^{\prime}(0)\right|^{2}\left(f^{\mu}\right)\right)(P+1) \bar{Q} \\
+2 \overline{\left(f^{\mu *}\left(H_{h_{0}}\right)\right.}\left(\left|Q_{h_{0}}{ }^{\prime}(0)\right|^{2}\left(f^{\mu}\right)\right)(P+1) Q \\
\left.+4\left|Q_{h_{0}}{ }^{\prime}(0)\right|^{2}\left(f^{\mu}\right)\right)\left((P+1)^{2}+|Q|^{2}\right) .
\end{gathered}
$$


By equation (5.1) we find $Q=\left(1-|\mu|^{2}\right)^{-1 / 2} \mu$ and $P=\left(1-|\mu|^{2}\right)^{-1 / 2}-1$. So we can write equations (5.6) and (5.7) in terms of $\mu$. Set

$$
\delta(\mu)=f^{\mu *}\left(Q_{h_{0}}{ }^{\prime}(0)\right)=\frac{\partial \mu}{\partial t}\left(1-|\mu|^{2}\right)^{-1}
$$

and

$$
H(\mu)=f^{\mu *}\left(H_{h_{0}}\right) .
$$

$$
\frac{\partial^{2}(P+1)^{2}}{\partial t^{2}}=(P+1)^{2}\left(\left(2 \operatorname{Re}\left(H(\mu)\left(|\delta(\mu)|^{2}\right) \bar{\mu}\right)+4|\delta(\mu)|^{2}\right)-2|\delta(\mu)|^{2} .\right.
$$

Moreover since $f^{\mu}$ preserves the volume form, from [18] Lemma 4.1, we find

$$
\begin{gathered}
f^{\mu *}\left(K_{r}\right)=\left(1-|\mu|^{2}\right)^{-1 / 2}\left(K_{r}-\bar{\mu} L_{r}\right) \\
+r / 2\left(1-|\mu|^{2}\right)^{-1 / 2}\left(K_{0}-\bar{\mu} L_{0}\right)\left(\log \left(1-|\mu|^{2}\right)\right) I \\
+r\left(1-|\mu|^{2}\right)^{-1 / 2}\left(\rho^{2} d z d \bar{z}\right)^{-1 / 2}(\overline{\partial \mu}) I .
\end{gathered}
$$

Since $L_{r}=\overline{K_{-r}}$, and $\Delta=4 L_{1} K_{0}$, it follows that the operator $H(\mu)$ depends only on $\mu$ and its derivatives on the surface $R$; it does not explicitly depend on the chosen solution to the Beltrami equation.

Here $H(\mu)$ is the operator $H_{h_{0}}$, with $h_{0}$ the metric with the same volume form as $g$, and with Beltrami differential $\mu$.

So, by using equations (5.7) and (5.8) we prove the following

Theorem 5.12. Consider the equation

$$
\begin{aligned}
\frac{\partial^{2} \mu}{\partial t^{2}} & =\left(1-|\mu|^{2}\right) H(\mu)\left(|\delta(\mu)|^{2}\right) \\
& +2 \frac{\partial \log \left(1-|\mu|^{2}\right)}{\partial t} \frac{\partial \mu}{\partial t} \\
& +2\left|\frac{\partial \mu}{\partial t}\right|^{2}\left(1-|\mu|^{2}\right)^{-1} \mu
\end{aligned}
$$

with the initial conditions $\mu(0)=0$ and $\frac{\partial \mu}{\partial t}(0)$ a fixed harmonic Beltrami differential in $(R, g)$. Then if $\mu(t)$ is the solution of the above equation, and $R(t)$ is the Riemann surface structure on $R$ such that $f^{\mu(t)}:(R, g) \rightarrow R((t)$ is holomorphic, then the curve given by $t \rightarrow$ Teichmüller classes of $R(t)$, is the Weil-Petersson geodesics starting at the class of $g$ with initial tangent vector $\frac{\partial \mu}{\partial t}(0)$. 
Note that equation (5.10) is equivalent to the system of first order equations:

$$
\left\{\begin{array}{l}
\frac{\partial \delta}{\partial t}=H(\mu)\left(|\delta|^{2}\right)-2 i(\operatorname{Im}(\delta \bar{\mu})) \delta \\
\frac{\partial \mu}{\partial t}=\delta\left(1-|\mu|^{2}\right)
\end{array}\right.
$$

with $\mu(0)=0$ and $\delta(0)=\frac{\partial \mu}{\partial t}(0)$. Moreover, since $f^{\mu}$ preserves the volume form, then equation (5.5) implies that $\int_{R}\left|\delta\left(\mu_{t}\right)\right|^{2} d V o l_{g}$ is independent on $t$, and it is the energy density of $\gamma$.

Remark 10. In other words, if $\mu_{t}$ is the solution of equation (5.10), then the (Lorentzian or $L^{2}$ ) lifting of the Weil-Petersson geodesics starting at $\mathrm{g}$ with initial tangent vector

$$
\frac{\partial \mu}{\partial t}(0) g+\overline{\frac{\partial \mu}{\partial t}(0) g}
$$

is given by

$$
\frac{\mu_{t}}{1-\left|\mu_{t}\right|^{2}} g+\left(\frac{1+\left|\mu_{t}\right|^{2}}{1-\left|\mu_{t}\right|^{2}}\right) g+\frac{\overline{\mu_{t}}}{1-\left|\mu_{t}\right|^{2}} g
$$

\section{The general existence Theorem.}

In this last section we are going to show that, whenever $g$ and $h$ have strictly negative curvature, there exists a unique critical point of $T_{\partial}$ in any homotopy class containing an orientation preserving diffeomorphism. By using Lemma (2.1) ii) we may reduce to the case of maps homotopic to the identity. Let $\tau$ be the harmonic map from $(R, h) \rightarrow(R, g)$ homotopic to the identity, so the identity is harmonic from $(R, h)$ to $\left(R, \tau^{*}(g)\right)$. Let $P, Q$ the Lorentzian exponential coordinates of $\tau^{*}(g)$ with respect to $h$. Set $\delta=1 / 2 \log (P+1)$, then the identity is harmonic from $\left(R, e^{2 \delta} h\right)$ to $\left(R, \tau^{*}(g)\right)$. Since the $P$ coordinate of $\tau^{*}(g)$ with respect to $e^{2 \delta} h$ is identically zero, it follows from Theorem 3.2 and from Lemma 2.1, that $\tau$ is a critical point of $T_{\partial}\left(e^{2 \delta} h, g\right)$ and $\phi_{0}=\tau^{-1}$ is critical with respect to $T_{\partial}\left(g, e^{2 \delta} h\right)$. Consider the product $\mathcal{D}_{0} \times \mathbf{R}$, and the subset $M \subseteq \mathcal{D}_{0} \times \mathbf{R}$, given by $M=\left\{(\phi, t)\right.$ such that $\left.\mathcal{E}\left(g, e^{2(1-t) \delta} h, \phi\right) \equiv 0\right\}$. Note that, by Lemma 2.2, if $(\phi, t)$ is in $\mathrm{M}$, then $e^{2(1-t) \delta} h$ has negative curvature. Let $\pi: M \rightarrow \mathbf{R}$ be the restriction of the natural projection. It follows from Lemma 2.3 and Lemma 2.4, together with the implicit function Theorem, that $M$ is a smooth submanifold of $\mathcal{D}_{0} \times \mathbf{R}$, and $\pi$ is a local diffeomorphism. The connected component containing $\left(\phi_{0}, 0\right)$ of the preimage through $\pi$ of the interval $[0,1]$, is a parameterized path $\gamma$ in $M$. We must show that $\gamma$ is 
defined in the whole interval $[0,1]$. If this were not the case, then $\gamma$ would be defined in an interval of the form $\left[0, t_{0}[\right.$, it is then sufficient to show the existence of a sequence $t_{n}$ converging to $t_{0}$, such that $\phi_{t_{n}}$ converges to a critical diffeomorphism $\phi_{t_{0}}$. Set $h_{t}=e^{2(1-t) \delta} h$ and $h_{t}^{\prime}=\phi_{t}{ }^{*}\left(h_{t}\right)$.

Lemma 6.1. We have $T_{\partial}\left(g, h_{t}, \phi_{t}\right), t \in\left[0, t_{0}[\right.$ is uniformly bounded.

Proof. Since each $\phi_{t}$ is critical, it follows that

$$
\frac{\partial T_{\partial}\left(g, h_{t}, \phi_{t}\right)}{\partial t} \leq \operatorname{Max}_{R}|\delta| T_{\partial}\left(g, h_{t}, \phi_{t}\right)
$$

so

$$
T_{\partial}\left(g, h_{t}, \phi_{t}\right) \leq T_{\partial}\left(g, h, \phi_{0}\right) e^{\operatorname{Max}_{R}|\delta|}
$$

We have that the metrics $g$ and $h_{t}^{\prime}$ are critical with respect to each other, let $m_{t}$ the medium point of the Lorentzian geodesics joining $g$ and $h^{\prime}{ }_{t}$. In the Lorentzian exponential coordinates centered at $m_{t}$, the geodesics is given by $P_{s}=s P, Q_{s}=s Q$, where $g$ has coordinates $\left(\begin{array}{c}P_{-1} \\ Q_{-1}\end{array}\right)$ and $h^{\prime}{ }_{t}$ has coordinates $\left(\begin{array}{c}P_{1} \\ Q_{1}\end{array}\right)$. (here $\left(\begin{array}{c}P \\ Q\end{array}\right)$ depends on $t$ but, for simplicity, we will drop the suffix). From above we find

$$
\begin{gathered}
1-P \geq|Q| \\
1+P \geq|Q|, \text { so } \\
-1<P<1, \text { and } 0 \leq|Q|<1 . \\
K_{m_{t}}=K_{g}\left((1-P)^{2}-|Q|^{2}\right)=K_{h^{\prime} t}\left((1+P)^{2}-|Q|^{2}\right)
\end{gathered}
$$

which gives

$$
4 K_{g}<K_{m_{t}}<0 .
$$

On the other hand, by using exponential coordinates $P^{*}, Q^{*}$ centered in $g$, it can be easily seen that

$$
d V o l\left(m_{t}\right)=1 / 4\left(d V o l(g)+d V o l\left(h_{t}^{\prime}\right)+2\left(P^{*}+1\right) d V o l_{g}\right.
$$

Let now $t_{n}$ be a sequence in $\left[0, t_{0}\left[\right.\right.$ converging to $t_{0}$. Set $\phi_{n}=\phi_{t_{n}}, h_{n}=h_{t_{n}}$, $h_{n}^{\prime}=h_{t_{n}}^{\prime}$, and $m_{n}=m_{t_{n}}$. 
Lemma 6.2. The energy of the map $\tilde{\phi}_{n}:\left(R, m_{n}\right) \rightarrow\left(R \times R, g \times h_{n}\right)$ is uniformly bounded.

Proof. Let us denote with Diag the diagonal map; we have

$$
\begin{aligned}
& E\left(m_{n}, g \times h_{n}, \tilde{\phi}_{n}\right)=E\left(m_{n}, g \times h_{n}{ }^{\prime}, \text { Diag }\right) \\
& =\int_{R}\left((1+P)^{2}+(1-P)^{2}+2|Q|^{2}\right) d V o_{m_{n}}
\end{aligned}
$$

which, because of estimates $(6.1)$ is bounded by $10 \mathrm{Vol}_{m_{t_{n}}}$. This in turns is uniformly bounded because of formula (6.4), Remark 2 and Lemma 6.1.

From this we immediately find

Lemima 6.3. The sequence of the Teichmüller classes of $m_{n}$ has a convergent subsequence.

Proof. By using the above Lemma we can argue as in [11].

If we act on the metrics $m_{n}$, and in the same time on the map $\tilde{\phi}_{n}$ with the group $\mathcal{D}_{0}$, we still find a sequence of minimal immersions with lagrangiam image, let us denote such immersions with $\Psi_{n}=\left(\Psi(1)_{n}, \Psi(2)_{n}\right)$. Note that $\Psi(1)_{n}$ and $\Psi(2)_{n}$ are $T_{\partial}$ critical with respect to the pairs of metrics $m_{n}, g$ and and $m_{n}, h_{n}$ respectively.

Remark 11. From formula (6.1) we can derive an $L^{\infty}$ bound of the differentials of $\Psi(1)_{n}$ and $\Psi(2)_{n}$ in terms of $L^{\infty}$ bounds of $m_{n}$.

We need to show that a subsequence of $m_{n}$ converges up to elements in $\mathcal{D}_{0}$. To do this we will use an easy version of Cheeger-Gromov compactness Theorem, see [9]. So it is necessary to have a bound on the curvatures, on the total volumes (which are immediate from equations (6.3), (6.4) and Lemma 6.1), and on the diameters. Let $m^{\prime}{ }_{n}$ be the metric conformal to $m_{n}$ with constant curvature -1 . We can assume that $m_{n}^{\prime}$ converges in $C^{\infty}$ norm, therefore to bound the diameter of $m_{n}$ it is sufficient to bound the volume forms of $m_{n}$.

Let us write in conformal coordinates

$$
m_{n}=\nu_{n}^{2}\left(\xi_{n}\right) d \xi_{n} d \bar{\xi}_{n}
$$


and

$$
m_{n}^{\prime}=\mu_{n}^{2}\left(\xi_{n}\right) d \xi_{n} d \overline{\xi_{n}}
$$

Lemma 6.4. There exists a uniform $L^{1}$ bound on $\log \left(\frac{\nu_{n}^{2}}{\mu_{n}^{2}}\right)$. Where $L^{1}$ is defined with respect to the measure of $m_{n}^{\prime}$. (Hence with respect to any fixed metric on $R$ ).

Proof. We have $\left|\log \left(\frac{\nu_{n}^{2}}{\mu_{n}^{2}}\right)\right| \leq \frac{\nu_{n}^{2}}{\mu_{n}{ }^{2}}+\frac{\mu_{n}^{2}}{\nu_{n}{ }^{2}}$. Formula (6.4) gives an $L^{1}$ bound on $\frac{\nu_{n}^{2}}{\mu_{n}^{2}}$. By Lemma 2.2 we have the equations

$$
\begin{gathered}
\partial_{n} \bar{\partial}_{n} \log \nu_{n}^{2}=-(1 / 2) K_{g}\left(\Psi(1)_{n}\right) \operatorname{det}\left(\operatorname{Jac}\left(\Psi(1)_{n}\right)\right)(i / 2) d \xi_{n} \wedge d \bar{\xi}_{n} \\
\partial_{n} \bar{\partial}_{n} \log \mu_{n}{ }^{2} d \xi_{n} \wedge d \bar{\xi}_{n}=\left(\frac{\mu_{n}^{2}}{2}\right)(i / 2) d \xi_{n} \wedge d \overline{\xi_{n}}
\end{gathered}
$$

For any positive constant $C$, we define the set $S_{C}=\left\{z \in R: \log \left(\frac{\nu_{n}^{2}}{\mu_{n}{ }^{2}}\right)<\right.$ $-C\}$. Since the curvature of $g$ is uniformly bounded, by using formulas (6.1), and (6.6), we see that, for large enough $C$ (independent on $n$ ) the function $\Delta_{n}\left(\log \left(\frac{\nu_{n}^{2}}{\mu_{n}^{2}}\right)\right)$ is negative on $S_{C}$, on the other hand, whenever $S_{C}$ is nonempty, the minimum of $\log \left(\frac{\nu_{n}^{2}}{\mu_{n}^{2}}\right)$ on $\overline{S_{C}}$ is in $S_{C}$, this yields a contradiction. Note that, we not only found an $L^{1}$ bound on $\left|\log \left(\frac{\nu_{n}{ }^{2}}{\mu_{n}{ }^{2}}\right)\right|$, but also an $L^{\infty}$ lower bound on $\log \left(\frac{\nu_{n}^{2}}{\mu_{n}{ }^{2}}\right)$.

To obtain an $L^{\infty}$ upper bound on $\left|\log \left(\frac{\nu_{n}^{2}}{\mu_{n}^{2}}\right)\right|$ we will use a so-called Wente estimate, see [2], applied to a relatively compact neighborhood $\Omega$ of a fundamental domain of $R$ in its universal covering space $D$. For simplicity any function on $R$ and its lift to $D$ will be denoted with the same symbol.

Lemma 6.5. There is an $L^{\infty}$ uniform bound on $\left|\log \left(\frac{\nu_{n}}{\mu_{n}}\right)\right|$.

Proof. Since $\mu_{n}$ converges and (by the proof of Lemma 6.4) the function $\log \left(\frac{\nu_{n}}{\mu_{n}}\right)$ has an $L^{\infty}$ lower bound, we only need to bound $\log \left(\nu_{n}{ }^{2}\right)$. Let us write $\log \left(\nu_{n}\right)=\tau_{n}+\eta_{n}$, where $\tau_{n}$ is harmonic on $\Omega$ and coincides with $\nu_{n}$ on $\partial \Omega$, whereas

$$
\partial_{n} \bar{\partial}_{n} \log \eta_{n}^{2}=-1 / 2 K_{g}\left(\Psi(1)_{n}\right) \operatorname{Jac}\left(\Psi(1)_{n}\right)
$$


Since the right hand side of (6.7) has a determinant form and the conformal structure of $m_{n}$ converges, we can apply Theorem 1.2 in [2], to obtain the estimate

$$
\left|\eta_{n}\right|_{L^{\infty}} \leq C\left|K_{g}\right|_{L^{\infty}}\left|d \Psi(1)_{n}\right|_{L^{2}(\Omega)}
$$

where $C$ is a positive constant independent on $n$, and the $L^{2}$ norm is computed with respect to the volume form of $m_{n}{ }^{\prime}$. Lemma 6.2 will give a uniform $L^{\infty}$ bound on $\eta_{n}$. Since $\tau_{n}$ is harmonic, then we can estimate its $L^{\infty}$ norm in terms of its $L^{1}$ norm on a domain $\Omega^{\prime}$ slightly smaller then $\Omega$. Now the Lemma follows from Lemma 6.4.

Similar Lemmas can be proved for the sequence $\Psi(2)_{n}$. By Remark (11) and Lemma 6.5 we derive an $L^{\infty}$ uniform bound on $d \Psi_{n}$. (All the bounds have to be considered with respect to the convergent sequence of hyperbolic metric, or, which is the same, with respect to a fixed metric). By using equation (6.6) we find an $L^{\infty}$ bound on the second derivative of $d V o l_{m_{n}}$. By Sobolev inequality in dimension 2, we obtain that (a subsequence of) $m_{n}$ converges to a metric $m$ in $C^{1, \alpha}$ (whereas ${m^{\prime}}_{n} \rightarrow m^{\prime}$ in norm $C^{\infty}$ ) and $\Psi_{n} \rightarrow \Psi$ in $C^{0, \alpha}$. Let us show that $\Psi$ is an immersion. To do this we will compare the metric $m_{n}$ with the metric $p_{n}$ which is the pull-back via $\Psi_{n}$ of the metric on $(R * R)_{n}$, we have

$$
\begin{gathered}
p_{n}=\frac{K_{m_{n}} \nu_{n}^{2} d \xi_{n} d \overline{\xi_{n}}}{\sqrt{K_{g} K_{h}}}\left\{\frac{1}{(P+1)^{2}-|Q|^{2}}\left((P+1) Q+\left((P+1)^{2}+|Q|^{2}\right)\right)\right\} \\
+\frac{K_{m_{n}} \nu_{n}^{2} d \xi_{n} d \overline{\xi_{n}}}{\sqrt{K_{g} K_{h}}} \frac{1}{(P+1)^{2}-|Q|^{2}}(P+1) \bar{Q} \\
+\frac{K_{m_{n}} \nu_{n}^{2} d \xi_{n} d \overline{\xi_{n}}}{\sqrt{K_{g} K_{h}}}\left\{\frac{1}{(1-P)^{2}-|Q|^{2}}\left(-(1-P) Q+\left((1-P)^{2}+|Q|^{2}\right)\right)\right\} \\
+\frac{K_{m_{n}} \nu_{n}^{2} d \xi_{n} d \overline{\xi_{n}}}{\sqrt{K_{g} K_{h}}}\left\{\frac{-1}{\left.(1-P)^{2}-|Q|^{2}\right)}(1-P) \bar{Q}\right\} .
\end{gathered}
$$

By a tedious but elementary computation using (6.1) we find

$$
\begin{gathered}
p_{n}=\frac{\nu_{n}^{2} d \xi_{n} d \overline{\xi_{n}}}{\sqrt{K_{g} K_{h}}}\left\{\left(K_{h}(1+P)-K_{g}(1-P)\right) \bar{Q}\right\} \\
+\frac{\nu_{n}^{2} d \xi_{n} d \overline{\xi_{n}}}{\sqrt{K_{g} K_{h}}} 2\left((1+P)^{2} K_{g}+|Q|^{2} K_{h}\right)
\end{gathered}
$$




$$
+\left\{\frac{\nu_{n}{ }^{2} d \xi_{n} d \bar{\xi}_{n}}{\sqrt{K_{g} K_{h}}}\left(K_{h}(1+P)-K_{g}(1-P)\right) Q .\right\}
$$

Therefore we get

$$
d V o l_{p_{n}}=2\left(\left(1-P^{2}\right)+|Q|^{2}\right) d V o l_{m_{n}}
$$

Since the curvature of $h_{n}$ is bounded away from zero, from the above formulas we derive the following

Lemma 6.6. The metrics $m_{n}$ and $p_{n}$ are uniformly comparable i.e. there exist positive constants $C_{1}$ and $C_{2}$, such that

$$
C_{1} m_{n} \leq p_{n} \leq C_{2} m_{n}
$$

It follows that $\Psi$ is an immersion.

Since $\Psi$ is an immersion then (by using notations of Remark 3) $\mathcal{H}_{1}\left(\Psi_{n}\right)$ and $\mathcal{L}_{1}\left(\Psi_{n}\right)$ as well as $\mathcal{H}_{2}\left(\Psi_{n}\right)$ and $\mathcal{L}_{2}\left(\Psi_{n}\right)$ do not have common zeros. Moreover since the maps $\Psi_{n}$ have lagrangian image, we have $\mathcal{H}_{1}\left(\Psi_{n}\right)=\mathcal{L}_{1}\left(\Psi_{n}\right)$ and $\mathcal{H}_{2}\left(\Psi_{n}\right)=\mathcal{L}_{2}\left(\Psi_{n}\right)$. Let us now prove that $\Psi_{n}$ converges to $\Psi$ in $C^{\infty}$ norm. The maps $\Psi_{n}:\left(R, p_{n}\right) \rightarrow(R * R)_{n}$ are minimal immersions with lagrangian image, then they satisfy equation (3.2), which, because of Lemma 6.6 , is a uniformly elliptic equation in divergence form. By standard estimates, see for example [4], and by formulas (6.1), we find a uniform $C^{1, \alpha}$ estimate on $\Psi_{n}$. Now by bootstrapping the equations (3.2) and (6.6) we conclude that $\Psi_{n}$ converges $C^{\infty}$ to $\Psi$. So $\Psi$ is a minimal immersion with lagrangian image To conclude we need to show that the components $\Psi(1)$ and $\Psi(2)$ of $\Psi$ are diffeomorphisms. Since they are limits of orientation preserving diffeomorphisms, then they have non negative jacobian determinants, moreover, $\mathcal{H}_{1}=\mathcal{H}_{2}$ and $\mathcal{L}_{1}=\mathcal{L}_{2}$, and since $\Psi$ is an immersion $\mathcal{H}_{1}$ and $\mathcal{L}_{1}$ as well as $\mathcal{H}_{2}$ and $\mathcal{L}_{2}$ do not have common zeros. If $p$ is a zero of the jacobian of, say $\Psi(1)$, then $\mathcal{H}_{1}$ and $\mathcal{L}_{1}$ do not vanish at $p$. If we let $l=\log \left(\frac{\mathcal{H}_{1}}{\mathcal{L}_{1}}\right)$ and $J^{*}=\mathcal{H}_{1}-\mathcal{L}_{1}$ we have that, near the point $p, J^{*}=f l$ where $f$ is a smooth function. Moreover, we already observed that $J^{*} \geq 0$, that is $l \geq 0$.

$$
|\Delta(l)| \leq C(|l|+|d(l)|) \text { near } p
$$

where $C$ is a positive constant. From above, by using a standard Harnack inequality see [5], we would conclude that $l$ vanishes in a neighborhood of $p$. Since $\Psi(1)$ and $\Psi(2)$ are non constant, it follows that they are orientation preserving diffeomorphism. Since the jacobians of $\Psi(1)$ and $\Psi(2)$ are 
bounded away from 0 , it follows that $\phi_{n}=\Psi(2)_{n} \circ \Psi(1)_{n}{ }^{-1}$ converges $C^{\infty}$ to $\phi_{t_{0}}=\Psi(2) \circ \Psi(1)^{-1}$. Hence $\phi$ is a $T_{\partial}$ critical map with respect to the metrics $\mathrm{g}$ and $h_{t_{0}}$. We are left to prove uniqueness. Let $\lambda$ and $\mu$ be smooth functions such that $e^{\lambda} g$ and $e^{\mu} h$ have constant curvature -1 . For a small positive $\varepsilon$ and for $t \in(-\varepsilon, 1+\varepsilon)$ we have that $e^{t \lambda} g$ and $e^{t \mu} h$ have negative curvature. Consider the set

$$
S=\left\{(t, \phi): \quad \phi \text { is critical with respect to } T_{\partial}\left(e^{t \lambda} g, e^{t \mu} h\right)\right\} .
$$

It can be proved as above that the projection map $S \rightarrow(-\varepsilon, 1+\varepsilon)$ is a covering. Since the fiber over $t=1$ is a single point, we conclude. We have then proved that Theorem 4.2 is valid for metrics with negative variable curvature as well, that is

Theorem 6.7. Let $g$ and $h$ be hermitian metrics on $R$, both with strictly negative curvature, then

(i) Suppose an homotopy class of maps of $R$ into itself contains an antiholomorphic non constant map, then there is only one such map and it is the unique critical point of $T_{\partial}$ in the class.

(ii) Suppose an homotopy class contains the constants, then these are the only critical points of $T_{\partial}$ in the class.

(iii) Suppose an homotopy class contains an orientation preserving diffeomorphism, then there exists a unique critical point of $T_{\partial}$ in the class. Such point is a diffeomorphism preserving the curvature forms.

(iv) In any other homotopy class there is no critical points of $T_{\partial}$.

If we denote by $\mathcal{M}_{-}$the subset of $\mathcal{M}$ given by metrics of negative curvature, we can state the above Theorem in the following geometric form

Theorem 6.8. Any two points in $\mathcal{M}_{-} / \mathcal{D}_{0}$ can be joined by a unique Lorentzian geodesics.

\section{Appendix.}

In this appendix we want to derive formula (3.2) and the Bochner formula (3.3). Set $a=\sqrt{-K_{g}}$ and $b=\sqrt{-K_{h}}$, then the Dirichlet energy of the map $\Psi$ is given

$$
E(\Psi)=\int_{R}\left(\frac{a}{b} \rho^{2}\left|d \Psi_{1}\right|^{2}+\frac{b}{a} \sigma^{2}\left|d \Psi_{2}\right|^{2}\right)(i / 2) d \zeta \wedge \overline{d \zeta} .
$$


Let $\Psi_{t}$ be a variation of $\Psi$, then the first derivative evaluated at $t=0$ is given by twice the real part of

$$
\begin{gathered}
\int_{R}\left(\frac{a}{b} \rho^{2}\left|d \Psi_{1}\right|^{2}\left(\partial_{z}(\log (a))+\partial_{z}\left(\log \left(\rho^{2}\right)\right)\right) \Psi_{1}{ }^{\prime}\right)(i / 2) d \zeta \wedge d \bar{\zeta} \\
-\int_{R}\left(\frac{b}{a} \sigma^{2}\left|d \Psi_{2}\right|^{2}\left(\partial_{z}(\log (a))\right){\Psi_{1}}^{\prime}\right)(i / 2) d \zeta \wedge d \bar{\zeta} \\
\quad+\int_{R} \frac{a}{b} \rho^{2}\left(\bar{\partial} \Psi_{1}{ }^{\prime} \partial \bar{\Psi}_{1}+\partial \Psi_{1}{ }^{\prime} \bar{\partial} \bar{\Psi}_{1}\right)(i / 2) d \zeta \wedge d \bar{\zeta}
\end{gathered}
$$

plus the analogue term in $\Psi_{2}^{\prime}$.

By integration by parts, formula (7.1) equals to

$$
\begin{gathered}
\int_{R}\left(\frac{a}{b} \rho^{2}\left|d \Psi_{1}\right|^{2}\left(\partial_{z}(\log (a))+\partial_{z}\left(\log \left(\rho^{2}\right)\right)\right) \Psi_{1}{ }^{\prime}\right)(i / 2) d \zeta \wedge d \bar{\zeta} \\
-\int_{R}\left(\frac{b}{a} \sigma^{2}\left|d \Psi_{2}\right|^{2}\left(\partial_{z}(\log (a))\right) \Psi_{1}^{\prime}\right)(i / 2) d \zeta \wedge d \bar{\zeta} \\
-\int_{R}\left(\bar{\partial}_{\zeta}\left(\frac{a}{b} \rho^{2} \partial \bar{\Psi}_{1}\right)+\partial_{\zeta}\left(\frac{a}{b} \rho^{2} \bar{\partial} \bar{\Psi}_{1}\right)\right)(i / 2) d \zeta \wedge d \bar{\zeta}
\end{gathered}
$$

Performing the differentiation in the above formula and collecting the term $\frac{a}{b} \rho^{2}$ we find the first equation of the Euler-Lagrange system, i.e.

$$
\left\{\begin{array}{l}
\bar{\partial} \partial \Psi_{1}+\partial_{z}\left(\log \rho^{2}\right) \bar{\partial} \Psi_{1} \partial \Psi_{1}-1 / 2\left(\bar{\partial} \Psi_{1} \partial_{\zeta} A_{1}+\partial \Psi_{1} \bar{\partial}_{\zeta} A_{1}\right) \\
=1 / 2\left(\bar{\partial}_{z}(\log (a))\left(\left|\partial \Psi_{1}\right|^{2}-\frac{b^{2}}{a^{2}}{\frac{\sigma^{2}}{\rho^{2}}}^{2}\left|\partial \Psi_{2}\right|^{2}\right)\right)
\end{array}\right.
$$

The same equation holds for $\Psi_{2}$. Notice that if $\Psi$ is minimal with lagrangian image, then the right hand side of the above equation vanishes, and we find equation (3.2).

In this case we derive a Bochner formula.

$$
\begin{gathered}
\left.\partial_{\zeta} \bar{\partial}_{\zeta}\left(\log \left(\rho^{2}\left|\partial \Psi_{1}\right|^{2}\right)\right)=\partial_{\zeta} \bar{\partial}_{\zeta}\left(\log \left(\rho^{2}\right)\right)+\partial_{\zeta} \bar{\partial}_{\zeta} \log \left(\partial \Psi_{1}\right)+\partial_{\zeta} \bar{\partial}_{\zeta} \log \left(\bar{\partial} \bar{\Psi}_{1}\right)\right)= \\
\bar{\partial}_{\zeta}\left(\partial_{z}\left(\log \left(\rho^{2}\right)\right) \partial \Psi_{1}+\bar{\partial}_{z}\left(\log \left(\rho^{2}\right)\right) \partial \bar{\Psi}_{1}\right)+\partial_{\zeta}\left(\frac{\partial \bar{\partial} \Psi_{1}}{\partial \Psi_{1}}\right)+\bar{\partial}_{\zeta}\left(\frac{\partial \bar{\partial} \bar{\Psi}_{1}}{\bar{\partial} \bar{\Psi}_{1}}\right)
\end{gathered}
$$

By using (3.2) we find 


$$
\begin{aligned}
\partial_{\zeta} \bar{\partial}_{\zeta}\left(\log \left(\rho^{2}\left|\partial \Psi_{1}\right|^{2}\right)\right) & =\bar{\partial}_{\zeta}\left(\partial_{z}\left(\log \left(\rho^{2}\right) \partial \Psi_{1}\right)-\partial_{\zeta}\left(\partial_{z}\left(\log \left(\rho^{2}\right) \bar{\partial} \Psi_{1}\right)\right)\right) \\
& +1 / 2 \partial_{\zeta}\left(\bar{\partial}_{\zeta}+\frac{\bar{\partial} \Psi_{1}}{\partial \Psi_{1}} \partial_{\zeta}\right) A_{1} \\
+ & 1 / 2 \bar{\partial}_{\zeta}\left(\left(\partial_{\zeta}+\frac{\partial \bar{\Psi}_{1}}{\bar{\partial} \bar{\Psi}_{1}} \bar{\partial}_{\zeta}\right) A_{1}\right)
\end{aligned}
$$

By standard computations we finally get

$$
\begin{gathered}
\partial_{\zeta} \bar{\partial}_{\zeta}\left(\log \left(\rho^{2}\left|\partial \Psi_{1}\right|^{2}\right)\right)=\partial_{z} \bar{\partial}_{z} \log \left(\rho^{2}\right)\left(\left|\partial \Psi_{1}\right|^{2}-\left|\bar{\partial} \Psi_{1}\right|^{2}\right) \\
+1 / 2 \partial_{\zeta}\left(\bar{\partial}+\frac{\bar{\partial} \Psi_{1}}{\partial \Psi_{1}} \partial\right) A_{1} \\
+1 / 2 \bar{\partial}_{\zeta}\left(\partial+\frac{\partial \bar{\Psi}_{1}}{\bar{\partial} \bar{\Psi}_{1}} \bar{\partial}\right) A_{1}
\end{gathered}
$$

Similarly

$$
\begin{aligned}
& \partial_{\zeta} \bar{\partial}_{\zeta}\left(\log \left(\rho^{2}\left|\bar{\partial} \Psi_{1}\right|^{2}\right)\right)=-\partial_{z} \bar{\partial}_{z} \log \left(\rho^{2}\right)\left(\left|\partial \Psi_{1}\right|^{2}-\left|\bar{\partial} \Psi_{1}\right|^{2}\right) \\
& +1 / 2 \partial_{\zeta}\left(\bar{\partial}+\frac{\bar{\partial} \bar{\Psi}_{1}}{\partial \bar{\Psi}_{1}} \partial_{\zeta}\right) A_{1}+1 / 2 \bar{\partial}_{\zeta}\left(\partial+\frac{\partial_{\zeta} \Psi_{1}}{\bar{\partial} \Psi_{1}} \bar{\partial}_{\zeta}\right) A_{1}
\end{aligned}
$$

By subtraction we obtain formula (3.3).

\section{References.}

[1] L. V. Ahlfors Lectures on quasiconformal mappings, Van Nostand, New York, 1966.

[2] F. Bethuel- J. M. Ghidaglia, Improved regularity of solutions of elliptic equations involving Jacobians and applications, J. Math. Pures Appl. 72 (1993), 441-473.

[3] J. Eells - J. H. Sampson, Harmonic mappings on Riemannian manifolds, Amer. J. Math. 8 (1964), 109-160.

[4] D. Gilbarg- N. S. Trudinger, elliptic partial differential equations of second order, Springer-Verlag, 1977.

[5] E. Heinz, On certain non linear elliptic differential equations and univalent mappings, J. d'Anal. Math. 5 (1956-57), 197-272. 
[6] N. J. Hitchin, The self-duality equation on Riemann surfaces, Proc. London Math. Soc. 55 n.3 (1987), 59-126.

[7] J. Jost - X. W. Peng, Group Actions, Gauge transformations, and the calculus of variations, Math. Ann. 293 (1992), 595-621.

[8] D. Mumford A Remark on Mahler's compactness Theorem, Proc. Am. Math. Soc. 28 (1971), 288-294.

[9] S. Peters, Convergence of Riemannian manifolds, Compositio Mathematica, 62 (1987), 3-16.

[10] R. Schoen - S. T. Yau, On univalent harmonic maps between surfaces, Invent. Math. 44 (1978), 265-278.

[11] _ Existence of incompressible minimal surfaces and the topology of three dimensional manifolds with nonnegative scalar curvature, Ann. of Math. (2) 110 (1979). 127-142.

[12] $\mathrm{R}$. Schoen, The role of harmonic mappings in rigidity and deformation problems, In Complex geometry Proceedings of the Osaka international conference, edited by Gen Komatsu - Yusuke Sakane. 1993, 179-200.

[13] A. J. Tromba, Teichmüller theory in Riemannian geometry, Birkhauser Verlag, Basel-Boston-Berlin, 1992.

[14] M. Wolf, The Teichmüller theory of harmonic maps, J. Diff. Geometry, 29 (1989), 449-479.

[15] S. Wolpert, Chern forms and Riemann tensor for the moduli space of curves, Inv. Math. 85 (1986), 119-145.

[16] Geodesics length functions and the Nielsen problem, J. Diff. Geometry, 25 (1987), 275-295.

[17] $\longrightarrow$ The hyperbolic metric and the geometry of the universal curve, J. Diff. Geometry, 31 (1990), 417-472.

[18] The Bers embedding and the Weil-Petersson metric, Duke Math. J. 60 n.2 (1990), 497-507.

RECEIVEd FEBRUARY 2ND, 1995.

Dipartamento di Matematica

UNIVERSITÁ DI ROMA 2 TOR VERgana

VIA DELLA RICERCA SCIENTIFICA 
00133 RoMA, ITALY

E-MAIL ADDRESS: TRAPANI@MAT.UTOVRM.IT

AND

Dipartamento di Matematica

Universitá Di PAVIA

Via AbBiategrasso 215

27100 PAVIA, ITALY

E-MAIL ADDRESS: VALLI@DRAGON.IAN.PV.CNR.IT 